\title{
Cystic fibrosis airway secretions exhibit mucin hyperconcentration and increased osmotic pressure
}

\author{
Ashley G. Henderson,, ${ }^{1,2}$ Camille Ehre, ${ }^{1}$ Brian Button, ${ }^{1}$ Lubna H. Abdullah, ${ }^{1}$ \\ Li-Heng Cai, ${ }^{3}$ Margaret W. Leigh, 1,4 Genevieve C. DeMaria, ${ }^{1}$ Hiro Matsui,, ${ }^{1}$ Scott H. Donaldson, ${ }^{1}$ \\ C. William Davis, ${ }^{1}$ John K. Sheehan, ${ }^{1}$ Richard C. Boucher, ${ }^{1}$ and Mehmet Kesimer ${ }^{1,5}$ \\ ${ }^{1}$ Cystic Fibrosis/Pulmonary Research and Treatment Center, ${ }^{2}$ Department of Medicine, Division of Pulmonary/Critical Care, \\ ${ }^{3}$ Department of Chemistry, ${ }^{4}$ Department of Pediatrics, and ${ }^{5}$ Department of Pathology and Laboratory Medicine, \\ University of North Carolina, Chapel Hill, North Carolina, USA.
}

\begin{abstract}
The pathogenesis of mucoinfective lung disease in cystic fibrosis (CF) patients likely involves poor mucus clearance. A recent model of mucus clearance predicts that mucus flow depends on the relative mucin concentration of the mucus layer compared with that of the periciliary layer; however, mucin concentrations have been difficult to measure in CF secretions. Here, we have shown that the concentration of mucin in CF sputum is low when measured by immunologically based techniques, and mass spectrometric analyses of CF mucins revealed mucin cleavage at antibody recognition sites. Using physical size exclusion chromatography/differential refractometry (SEC/dRI) techniques, we determined that mucin concentrations in CF secretions were higher than those in normal secretions. Measurements of partial osmotic pressures revealed that the partial osmotic pressure of CF sputum and the retained mucus in excised CF lungs were substantially greater than the partial osmotic pressure of normal secretions. Our data reveal that mucin concentration cannot be accurately measured immunologically in proteolytically active CF secretions; mucins are hyperconcentrated in CF secretions; and CF secretion osmotic pressures predict mucus layer-dependent osmotic compression of the periciliary liquid layer in CF lungs. Consequently, mucin hypersecretion likely produces mucus stasis, which contributes to key infectious and inflammatory components of CF lung disease.
\end{abstract}

\section{Introduction}

Despite an in-depth understanding of the genetic and molecular basis of cystic fibrosis (CF), the pathogenesis of CF mucoinfective lung disease remains unclear (1). The mechanical clearance of bacteria from the lungs is mediated by cilia- and cough-dependent clearance of mucus $(2,3)$, and there are data suggesting that defects in mucus clearance occur early in CF pathogenesis $(4,5)$. Additionally, there are reports of an impairment of antimicrobial killing in the surface liquid of CF airways that is potentially $\mathrm{pH}$ dependent $(6,7)$. However, based on the likelihood that bacterial selection will quickly generate organisms resistant to airway surface liquid (ASL) killing mechanisms, it is probable that mechanical clearance of bacteria is central to lung defense (8).

The mucus that constitutes the mobile mucus layer covering human airways is a complex mixture of mucins, globular proteins, antimicrobial proteins and peptides, salts, and water. The gel-forming mucins MUC5B and MUC5AC, however, are the major macromolecular contributors to the non-Newtonian properties of mucus responsible for transport of this layer $(2,9-11)$.

Authorship note: Ashley G. Henderson and Camille Ehre contributed equally to this work. Richard C. Boucher and Mehmet Kesimer are co-senior authors. John K. Sheehan is deceased.

Conflict of interest: The authors have declared that no conflict of interest exists.

Note regarding evaluation of this manuscript: Manuscripts authored by scientists associated with Duke University, The University of North Carolina at Chapel Hill, Duke-NUS, and the Sanford-Burnham Medical Research Institute are handled not by members of the editorial board but rather by the science editors, who consult with selected external editors and reviewers.

Citation for this article: J Clin Invest. 2014;124(7):3047-3060. doi:10.1172/JCI73469
Secreted gel-forming mucins are large, polymeric, glycosylated proteins, with molecular weights ranging from approximately 2 to $50 \mathrm{MDa}$, and up to $80 \%$ of their mass is carbohydrate. Of the secreted mucins, MUC5AC and MUC5B are the dominant secreted mucins in both normal and diseased human airways $(12,13)$. Recent data have suggested that the transportability of the mucus layer is heavily dependent on the concentration of mucins in the mucus layer, which in overlap (semi-dilute) conditions theoretically generates partial (i.e., mucin-dominated) osmotic pressures that are a function of the mucin concentration to the power of 2.5 (2). In $\mathrm{CF}$, an increase in mucin concentration and partial osmotic pressure would be predicted to result from a decrease in airway surface liquid volume and/or an increase in mucin secretion, slowing mucus clearance and producing mucostasis (14).

However, few studies have measured MUC5AC and MUC5B mucin concentrations in normal or CF airway secretions $(10,15-17)$. In one recent report, Henke et al. suggested that there may be no difference, or a dramatic decrease rather than increase, in the concentrations of the secreted mucins MUC5AC and MUC5B in CF mucus depending on Pseudomonas aeruginosa ( $P$. aeruginosa) infection status $(10,16-18)$. Importantly, Henke et al. used solely antibody-dependent analyses of agarose gel Western blots as the basis for mucin quantitation in their studies. Thus, the reports of Henke et al. suggest that CF mucus is dominated by macromolecules other than mucins, e.g., DNA, that determine its biophysical properties and modulate its transport $(17,19)$.

In the present study, we sought to measure mucin concentrations in normal and CF pulmonary secretions using a combination of techniques. Based on previous reports of mucin proteolysis 
A

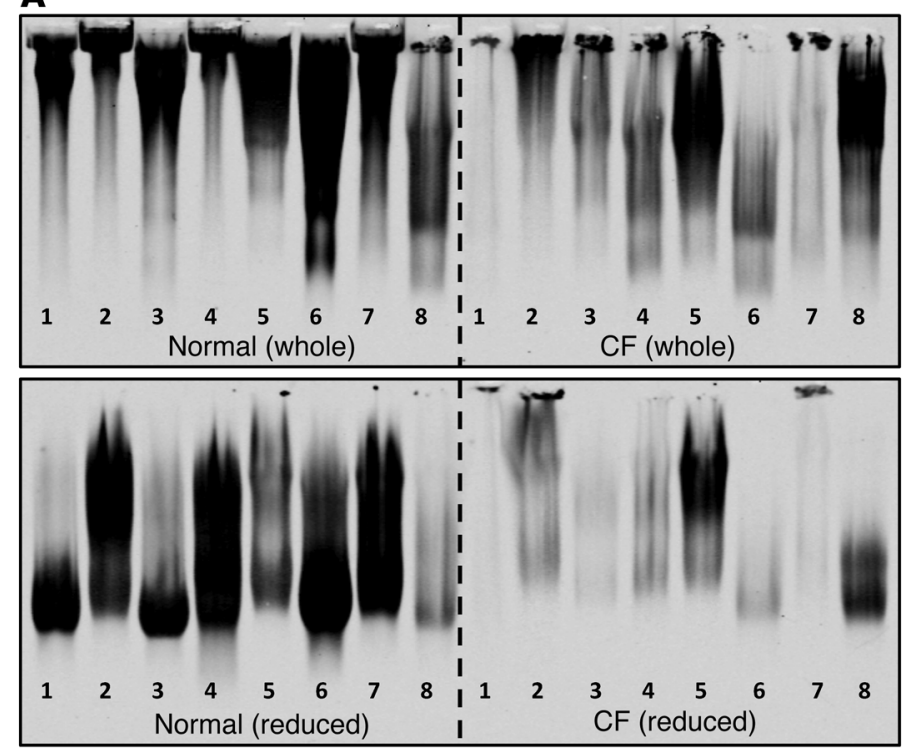

B

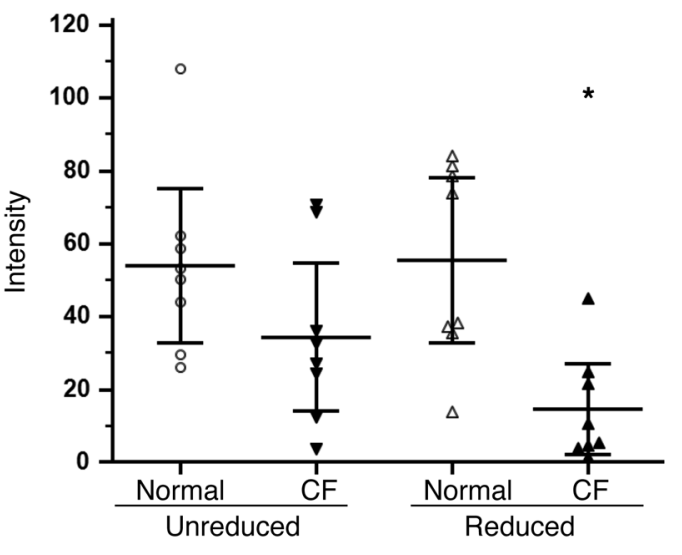

Figure 1

Mucin (MUC5B) levels in CF sputum as monitored by immunoblotting. (A) Agarose gel electrophoresis of sputum from 8 normal and CF patients; whole or reduced samples were probed with a monoclonal MUC5B antibody (EU-MUC5Ba) prior to loading. The integrated intensities of the each lane were quantitated and scatter plotted for comparison (B). Mean and standard error values are indicated by major (mean) and minor (SEM) horizontal bars. The independent samples $t$ test was used to determine changes between normal and CF sputum in values for whole $(P=0.13)$ and reduced ( ${ }^{\star} P=0.0023$ ) conditions, indicating significant loss of MUC5B reactivity after reduction.

in airway secretions $(10,18,20)$ and our own preliminary data, we first investigated the hypothesis that immunologically based quantitation methods are inaccurate for mucin quantitation in CF airway secretions due to proteolytic degradation of exposed epitopes within mucin molecules (18). Second, we compared physical versus immunologic methods for mucin quantitation using a combination of size exclusion chromatography coupled to differential refractive index measurement (SEC/dRI) techniques to measure mucin concentrations in samples from normal and CF subjects and from cell cultures. Third, we asked whether an increase in mucin concentration in CF secretions may, in part, reflect an increase in airway epithelial mucin gene transcription and/or mucin secretory cell numbers. Finally, because the partial osmotic pressure of mucus may be predictive of mucus flow versus no flow, a novel technique for measuring the partial osmotic pressure of mucus was developed and applied to normal and CF samples (2).

\section{Results}

\section{Immunologic measurements of mucins in CF versus normal secretions and analyses of mucin epitope integrity}

Measurements of MUC5B in CF versus normal sputum. To quantitate MUC5B mucin concentrations in samples similar to those of Henke et al. (17), sputum samples were obtained from CF and normal subjects, solubilized in $\mathrm{GuHCl}$, and analyzed via agarose gel electrophoresis under both whole (unreduced) and reduced conditions. As seen in Figure 1, whole CF sputum exhibited an approximately $40 \%$ reduction in MUC5B antibody immunoreactivity compared with that in whole normal sputum. There was a further substantial decrease in the MUC5B antibody immunoreactivity in all CF samples when these samples were reduced.
Measurements of mucins and lactoferrin in pediatric BAL. We also performed immunologic quantitation of mucins was in pediatric BAL. We hypothesized that all macromolecules secreted by airway epithelia and/or glands would be concentrated in CF mucus secretions harvested by BAL from pediatric patients. Accordingly, we analyzed BAL samples from non-CF disease control and CF subjects for lactoferrin, an index of gland antimicrobial protein secretion, and used immunologic techniques to analyze the secreted gel-forming airway mucins MUC5AC and MUC5B (Figure 2). As predicted, lactoferrin concentrations were increased in CF BAL samples as compared with those found in disease control samples (Figure 2A). In the non-CF disease controls, MUC5B was the dominant secreted mucin (Figure 2B). Like Henke et al. (17) and as shown in Figure 1, MUC5B levels appeared to be reduced in CF samples compared with normal samples when mucin quantitation was determined by immunodetection. We found that MUC5AC levels were not significantly lower in the CF samples.

\section{Protease effects on mucin concentrations and macromolecular mass measured by immunologic techniques in CF versus normal airway secretions}

Assessment of the effect of trypsin and buman neutrophil elastase on mucin immunoreactivity. CF sputum typically exhibits substantial quantities of free neutrophil elastase (NE) (21). To elucidate the effect of proteases including elastase on immunodetection of mucin, purified mucins were subjected to controlled proteolysis in vitro over a 5- to 240-minute time course. We measured the proteolytic effects on mucin antibody reactivity with 2 different antibodies. Probing with a polyclonal MUC5B antibody (MAN5BIII) revealed that trypsin treatment substantially reduced the antibody reactivity over the 240-minute incubation (Figure 3A). In compari- 


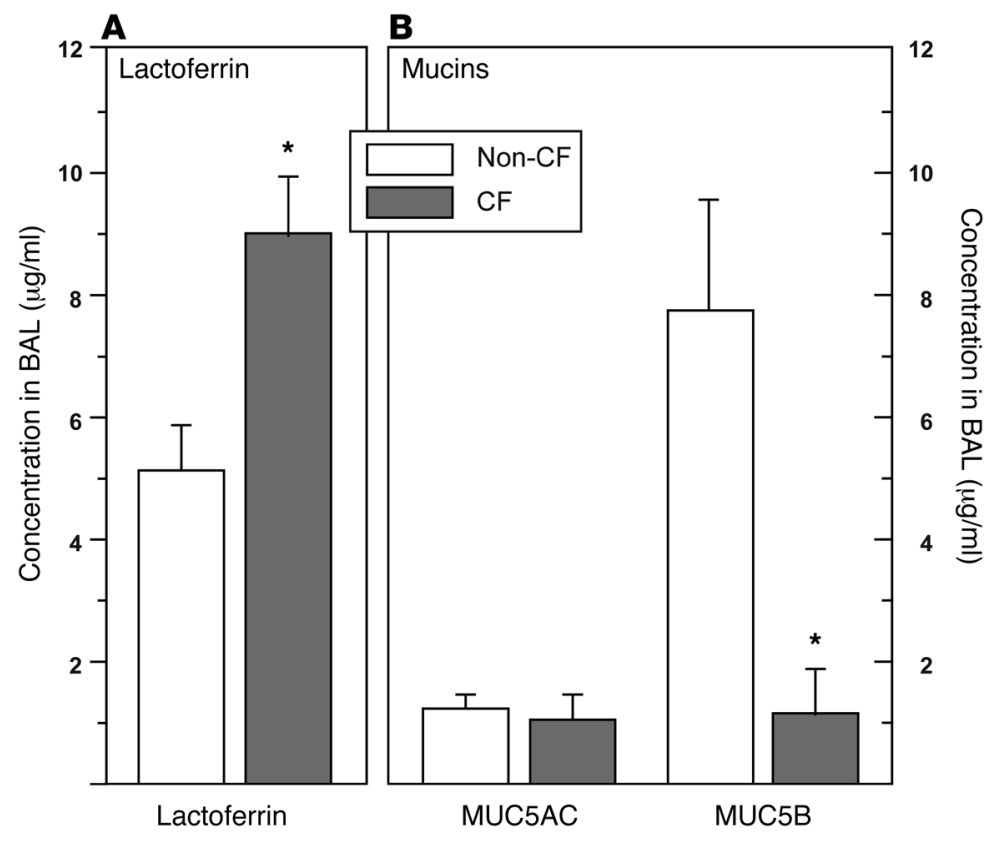

Figure 2

Lactoferrin (A) and polymeric mucin (B) levels in BAL fluid samples from children with CF or other disorders (non-CF disease controls). (A) Lactoferrin concentrations were increased in CF BAL samples as compared with those in disease control samples. In the non-CF disease controls, MUC5B was the dominant secreted mucin. MUC5B levels were reduced in CF versus normal samples when mucin quantitation was determined by immunodetection. MUC5AC levels were not significantly lower in CF samples. Data represent the mean \pm SEM $\left({ }^{\star} P<0.01\right)$.

son, incubation with NEs at concentrations mimicking those in CF sputum $(1 \mu \mathrm{g} / \mathrm{ml})(22)$ virtually abolished antibody reactivity within 5 minutes (Figure 3A). Studies of immunoreactivity using a monoclonal MUC5B antibody (EU-MUC5Ba) indicated that both elastase and trypsin abolished mucin detection after 20 minutes of exposure (Figure 3A).

Assessment of human NE on mucin molecular mass and integrity. Multiangle light scattering coupled to size exclusion chromatography (SEC/MALS) measurements to determine the macromolecular integrity of purified salivary MUC5B indicated that after $60 \mathrm{~min}-$ utes of elastase exposure, at least $80 \%$ to $90 \%$ of the mucins maintained a molecular weight of approximately $38 \mathrm{MDa}$ (Figure 3B), i.e., that typical of purified MUC5B $(23,24)$. A typical dRI trace (solid lines) and the molecular mass distribution (dotted lines) in the void $\left(\mathrm{V}_{\mathrm{o}}\right)$ volume region are shown in Figure $3 \mathrm{~B}$. The total mucin mass in the $V_{o}$ volume was monitored by $d R I$ and was slightly decreased from $28.4 \mu \mathrm{g}( \pm 1.2 \mu \mathrm{g})$ (blue line, PBS control) to $25.6 \mu \mathrm{g}( \pm 0.9 \mu \mathrm{g})$ after a 20 -minute elastase incubation (green line) and to $24.2 \mu \mathrm{g}( \pm 0.8 \mu \mathrm{g})$ after a 60 -minute elastase incubation (magenta line). Corresponding molecular mass calculations showed that control mucins exhibited an average molecular mass of approximately $38 \mathrm{MDa}( \pm 6.2 \%)$ (blue dotted line), which slightly increased to approximately $44 \mathrm{MDa}( \pm 4.2 \%)$ after $20 \mathrm{~min}-$ utes (green dotted line) and 60 minutes (magenta dotted line) of elastase incubation. These findings suggest that relatively short (up to 60-minute) incubation periods of NE with these samples did not affect the macromolecular integrity of the MUC5B macromolecule, despite dramatically affecting the antibody reactivity.
In parallel experiments, whole HBE mucus (2.5\%) was incubated with human NE and PBS. We performed cone and plate rheometry measurements and detected no differences in viscosity profiles for elastase versus PBS incubation over the same 60-minute time period (Supplemental Figure 2; supplemental material available online with this article; doi:10.1172/JCI73469DS1). These data also suggest that elastase did not affect the macromolecular properties of mucus responsible for key biophysical parameters, i.e., viscosity.

$P$. aeruginosa affects MUC5B antibody detection. Because $P$. aeruginosa has been reported to cause proteolytic degradation of mucins $(16,25)$, we next tested whether its presence is also a contributor to the proteolytic degradation of mucins in CF sputum samples. We tested antibody reactivity by comparing the biochemical and immunologic features of mucins in sterile human bronchial epithelial (HBE) mucus in sputum from a $P$. aeruginosa-infected CF patient and in sterile mucus of varying concentrations generated from HBE cultures with or without exposure to $P$. aeruginosa. Two different concentrations of HBE mucus $(2.5 \%$ approximating normal; $8 \%$ approximating $C F$ ) were incubated for 72 hours with a laboratory strain of $P$. aeruginosa (PAO1) or a mutant form of P. aeruginosa (PAO-JP1) (26), which lacks the lasI quorum-sensing gene that controls $P$. aeruginosa elastase gene expression and therefore expresses minimal elastase activity (27). After initial seeding, we observed by microscopy that the bacteria were growing as biofilms in $8 \%$ mucus, but not in $2.5 \%$ mucus, within 24 to 48 hours (not shown). Following the 72-hour culture interval, we investigated the immunologically detected integrity of the mucins in the HBE cell culture samples and CF sputum samples using agarose gel electrophoresis and immunoblotting (Figure 4). The top panel (Figure 4A) represents whole samples (no reduction prior to loading the agarose gel); the bottom panel (Figure 4B) represents the samples that were reduced with DTT prior to loading into the agarose gel.

Analyses of the whole (unreduced) samples demonstrated that the $P$. aeruginosa-infected CF sputum (Figure 4A, lane 1) migrated into the gel faster than did the sterile (non- $P$. aeruginosa-containing) HBE culture mucus (Figure 4A, lane 2). The PAO-JPI HBE culture (mutant $P$. aernginosa with minimal elastase; Figure 4A, lane 3 ) exhibited a profile similar to that of the sterile mucus (Figure 4A, lane 2). In contrast, we found that the addition of PAO1 to $8 \%$ mucus, which produced biofilms and elastase, was associated with the absence of detectable MUC5B (Figure 4A, lane 4). Exposure of PAO1 to $2.5 \%$ mucus, which did not support biofilm formation, was associated with decreased proteolysis compared with exposure of PAO1 to $8 \%$ mucus (Figure 4A, lane 5). Reduction accentuated the effects of proteolysis on the immunodetection of MUC5B, with only mutant $P$. aeruginosa (with minimal elastase) and normal HBE samples exhibiting detectable MUC5B after reduction (Figure 4B, lanes 2 and 3 ). As expected with reduction, these 2 lanes exhibited further migration into the gel after reduction. Even though the cell culture with PAO1 in $2.5 \%$ mucus did not form biofilms and hence maintained immunodetection before reduction, the presence of $P$. aeruginosa was sufficient to result in loss of immunodetection after reduction. 
A

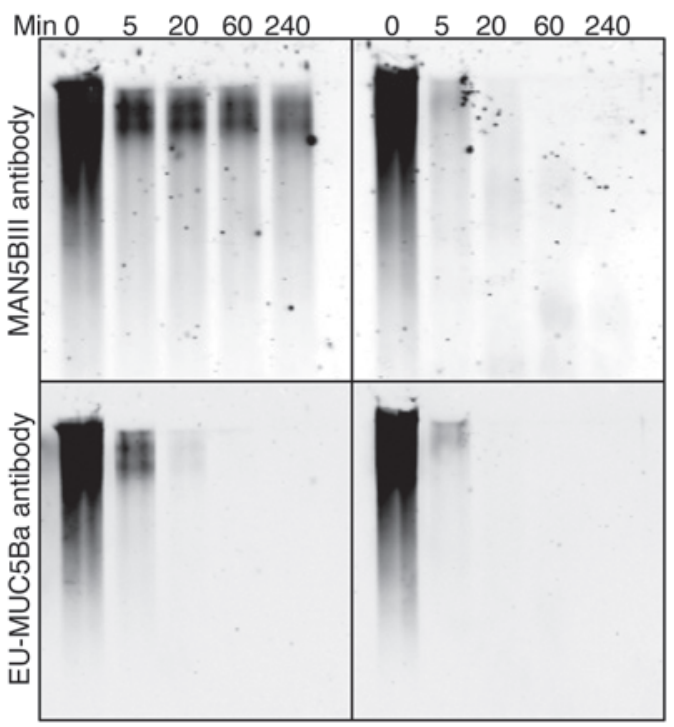

B

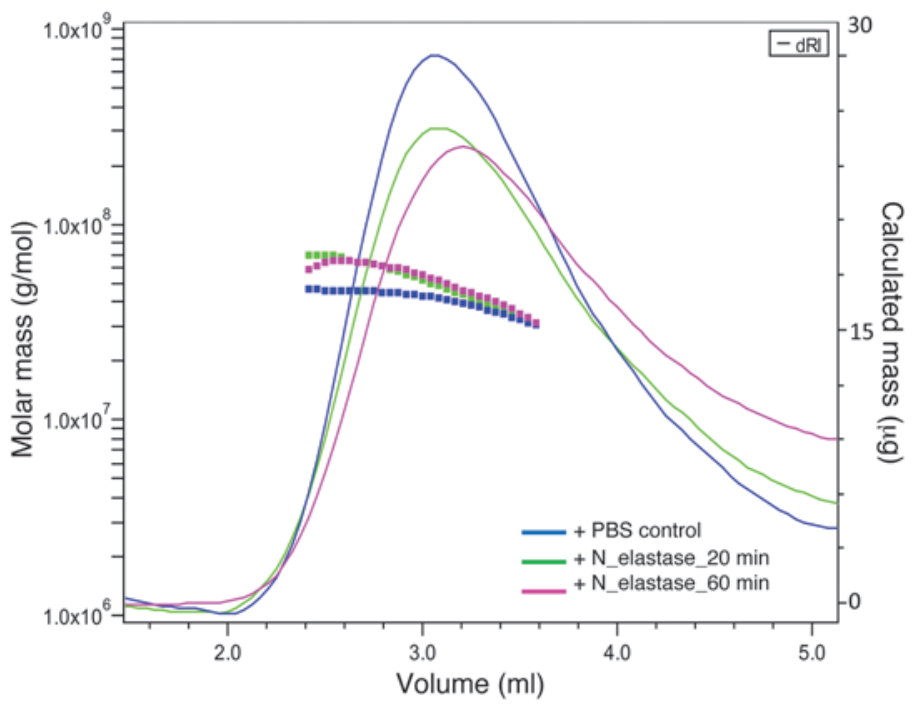

Figure 3

The effect of proteases on mucin epitopes and polymeric structure. (A) Isolated MUC5B $(100 \mu \mathrm{g})$ was incubated with either trypsin $(1 \mu \mathrm{g})$ or elastase $(1 \mu \mathrm{g})$ for increasing periods of time (5 minutes to 4 hours). Unreduced aliquots of the samples were subjected to agarose gel electrophoresis and probed with MAN5BIII or EU-MUC5Ba antibodies. MAN5BIII immunoreactivity was completely abolished by the NE within 5 minutes, while trypsin treatment substantially reduced the antibody reactivity. EU-MUC5Ba immunoreactivity was lost within 20 minutes of exposure to both trypsin and elastase. (B) An aliquot from the NE-treated samples was subjected to SEC/MALS/dRI measurements using a Sepharose CL-2B $(2 \times 5 \mathrm{ml})$ column to determine molecular weight (dotted lines) and sample concentrations (solid lines), respectively. The total mucin mass under the curve was slightly decreased from $28.4 \mu \mathrm{g}$ (blue line, PBS control) to $25.6 \mu \mathrm{g}$ after a 20 -minute elastase incubation (green line) and to $24.2 \mu \mathrm{g}$ after a 60-minute elastase incubation (magenta line). Molecular mass measurements were plotted across the mucin peak: control mucins had an average molecular mass of approximately $38 \mathrm{MDa}$ (blue dotted line) and had an average molecular mass of approximately $44 \mathrm{MDa}$ after 20 (green dotted line) and 60 (magenta dotted line) minutes of elastase incubation, indicating a slight increase in molecular weight.

Mass spectrometry demonstrates proteolytic cleavage of MUC5B in native $C F$ sputum and HBE cell culture samples containing $P$. aeruginosa. Analysis of the tryptic peptides from both normal sputum and $\mathrm{HBE}$ mucus indicated that the secreted mucins MUC5B and MUC5AC were the major molecules in the density gradient CL-2B void fractions with few other globular proteins detected (data not shown).

The tryptic peptide coverage we obtained for MUC5B isolated from normal induced sputum samples was extensive $(\sim 20 \%)$ and widely dispersed throughout the molecule (Figure 5). Analyses of MUC5B isolated from normal saliva revealed a similar comprehensive coverage (not shown). However, our analysis of the MUC5B mucin isolated from $\mathrm{CF}$ sputum revealed a much more restricted coverage $(-8 \%)$ of the molecule, with notable loss of coverage in the $\mathrm{C}$ terminus, Cys-rich regions, and especially in the $\mathrm{N}$ termini (Figure 5).

We next tested whether the presence of $P$. aeruginosa could reproduce this cleavage pattern of mucin molecules by comparing nontreated (sterile) with $P$. aeruginosa-treated $8 \%$ HBE mucus samples. The coverage of MUC5B isolated from the sterile mucus was complete and similar to that of normal sputum (20\%). In contrast, coverage of $P$. aeruginosa-treated CF-like mucus was also heavily restricted (8\%), and the pattern of loss of coverage was similar to that observed in CF sputum (Figure 5).

Separation and identification of the oligomeric state of gel-forming mucins in CF versus normal sputum by $C L-2 B$ chromatography

The oligomeric integrity of the mucin molecules in normal and CF samples was next characterized by CL-2B gel filtration chromatography and immunoblotting (Figure 6). In the normal samples, the whole (unreduced) MUC5AC and 5B molecules ran in the exclusion limit, i.e., $V_{0}$ fractions, of the CL-2B chromatographic column, as expected for airway mucins (ref. 28 and Figure 6, A and B). In contrast, reduced samples exhibited more included volume $\left(\mathrm{V}_{\text {inc }}\right)$ distributions for MUC5AC and MUC5B proteins in the column, as predicted for structures of lower molecular weight (Figure 6, A and B).

The analyses of whole (unreduced) CF samples revealed a relatively intact molecular size for MUC5AC. There was some evidence for increased penetration into the included volume for MUC5B, but the pattern was generally similar to that of MUC5B obtained from normal sputum (Figure 6, C and D). In contrast, our analysis of reduced CF mucus samples demonstrated 2 features that were strikingly different from those of the normal samples. First, reduction completely abolished antibody reactivity to MUC5AC (Figure 6C). Second, the size of immunoreactive MUC5B molecules after reduction was significantly smaller than that of the normal samples (Figure 6, B and D). For example, we observed no immunoreactivity of CF MUC5B between fractions 10-20 (Figure 6D), in which monomers were found in the normal samples (Figure 6B). These findings suggest that reduction revealed proteolytic cleavages of protein monomers in CF samples. Importantly, the integrity of mucin oligomers in CF sputum in the whole (i.e., unreduced) state suggests that disulfide bonds preserve the oligomeric forms of mucins and maintain key biophysical properties of mucins (Figure 6, C and D). 


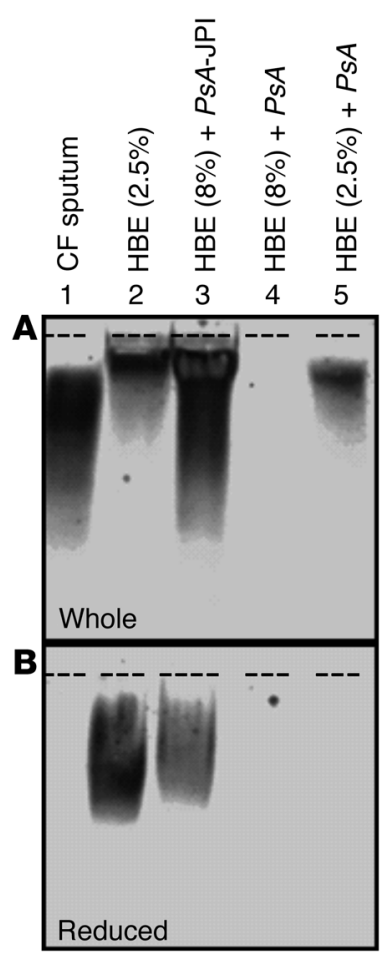

\section{Measuring gel-forming mucin gene expression in normal and $\mathrm{CF}$ lung tissues}

To test whether there was increased mucin synthesis by CF airway epithelia, total mRNA was extracted from normal $(n=14)$ and CF $(n=10)$ bronchi ex vivo, and mucin gene expression was measured via real-time PCR. Since small changes in Ct (threshold or inflection cycle, also called cross-point) lead to marked differences in fold changes, results are shown as the relative changes in cross-point $(\mathrm{Ct})$ values for MUC5AC and MUC5B mRNA levels. We found that both MUC5AC and MUC5B mRNA levels were increased in CF lungs as compared with the levels in non-CF lungs (Figure 7). Fold-change calculations by the $2^{\text {-average } \Delta \Delta \mathrm{Ct}}$ formula revealed a 4.5- and a 2.6-fold increase in CF for MUC5AC and $M U C 5 B$ gene expression, respectively. For this experiment, primers for MUC5AC and MUC5B were designed to exhibit comparable efficiency $(\sim 2)$. On average, MUC5AC cross-point values were lower than those for MUC5B, indicating that MUC5AC was the dominant mucin expressed in airway cell surfaces. Note that all "normal," non-CF lungs were subjected to mechanical ventilation for 1 to 3 days prior to dissection, which is known to stimulate mucin gene expression as well as absolute values in MUC5AC and MUC5B and their ratios (15). Hence, this intervention may have led to an underestimation of CF fold changes in this data set.

\section{Immunohistochemical detection of intracellular mucins in normal and $\mathrm{CF}$ airways}

Airway secretions stained with Alcian Blue-PAS (AB-PAS) revealed evidence for goblet cell hyperplasia in CF bronchi and bronchioles compared with that observed in normal airway epithelia (Figure 8A), as previously reported (15). In addition, intense AB-PAS staining was detected in the lumens of CF bronchi and bronchioles, but not in normal airways. MUC5AC and MUC5B immunostaining of CF airways confirmed the increase in airway epithelial goblet

\section{Figure 4}

Mucin (MUC5B) immunoblotting of samples with variable mucus concentration, with and without the presence of $P$. aeruginosa. (A) Unreduced (whole) samples. Lane 1 contains sputum collected from a representative CF patient infected with $P$. aeruginosa. Lane 2 contains normal $2.5 \%$ mucus cell culture as a control. Lane 3 contains $8 \%$ mucus cell culture with $P$. aeruginosa-JP1 ( $P$. aeruginosa lacking biofilm-forming capacity and lower elastase production). Lane 4 contains $8 \%$ cell culture, $8 \%$ mucus, and $P$. aeruginosa. Lane 5 contains $2.5 \%$ mucus cell culture with $P$. aeruginosa added to the culture. All samples in lanes 1-5 were not reduced prior to loading (whole samples). (B) Samples were reduced prior to loading, leading to a loss of antibody reactivity in $P$. aeruginosa-exposed samples. $P$. aeruginosa-JP1 samples (lanes 3 in $\mathbf{A}$ and $\mathbf{B}$ ) maintained some antibody reactivity, as this $P$. aeruginosa mutant exhibited much lower elastase production, suggesting that the $P$. aeruginosa proteolysis caused the absence of antibody staining in these gels. Origins of the loaded samples are indicated by the dashed lines. Gels shown are representative of at least 3 independent experiments. PsA, P. aeruginosa.

cells in CF airways (Figure 8B). The acellular material in the lumen stained less intensely than did the intracellular materials in the airway epithelial cells, consistent with proteolytic cleavage. In normal conducting airways, MUC5AC is mostly expressed in surface epithelium, while MUC5B is expressed in submucosal glands (29).

Direct comparison of immunologic versus biophysical measurements of newly secreted mucins added to CF airway supernatant mucopurulent material

To determine whether the apparent loss of immunoreactivity observed for mucins sampled from CF sputum could be reproduced in vitro, we treated HBE cultures for 72 hours with CF supernatant mucopurulent material (SMM) to replicate the mucin hypersecretory environment of CF (30) and then sequentially exposed the cultures to ATP $\gamma \mathrm{S}(100 \mu \mathrm{M}, 60$ minutes), followed by exposure to fractionated SMM on PBS for 2 hours (see Methods). We analyzed MUC5B in the secretions harvested from the cultures by agarose Western blotting (Figure 9A) or SEC/ dRI (Figure 9B). The apparent mucin concentration in the fractionated SMM-exposed samples measured by immunologic techniques was significantly decreased in comparison with that in the PBS samples (Figure 9A). However, analyses by SEC/dRI revealed that total mucin secreted in response to ATP $\gamma \mathrm{S}$ stimulus was similar in samples exposed to filtered SMM and PBS $(6 \pm 2 \mathrm{mg} / \mathrm{ml})$. The observation that total mucin concentrations, as measured by immunologic techniques, decreased when fractionated SMM was added to ATP $\gamma$ S-treated cultures, rather than not being changed as measured by physical techniques, strongly argues that the proteolytic activities in fractionated SMM prevented accurate measurements of mucin concentrations. Thus, a direct comparison of immunologic versus physical techniques in a controlled environment indicated that immunologic measurements were not accurate in CF-like environments.

\section{Mucin concentration in CF versus normal sputum as measured by a} physical (refractometry) approach

Based on the data shown in Figure 9 that biophysical measurements are required to accurately measure mucin concentrations in infected sputum, sputum samples from $10 \mathrm{CF}$ individuals (spontaneously produced) and 8 normal subjects (via induction) were collected, solubilized in $6 \mathrm{M} \mathrm{GuHCl}$, diluted in PBS with DNase 


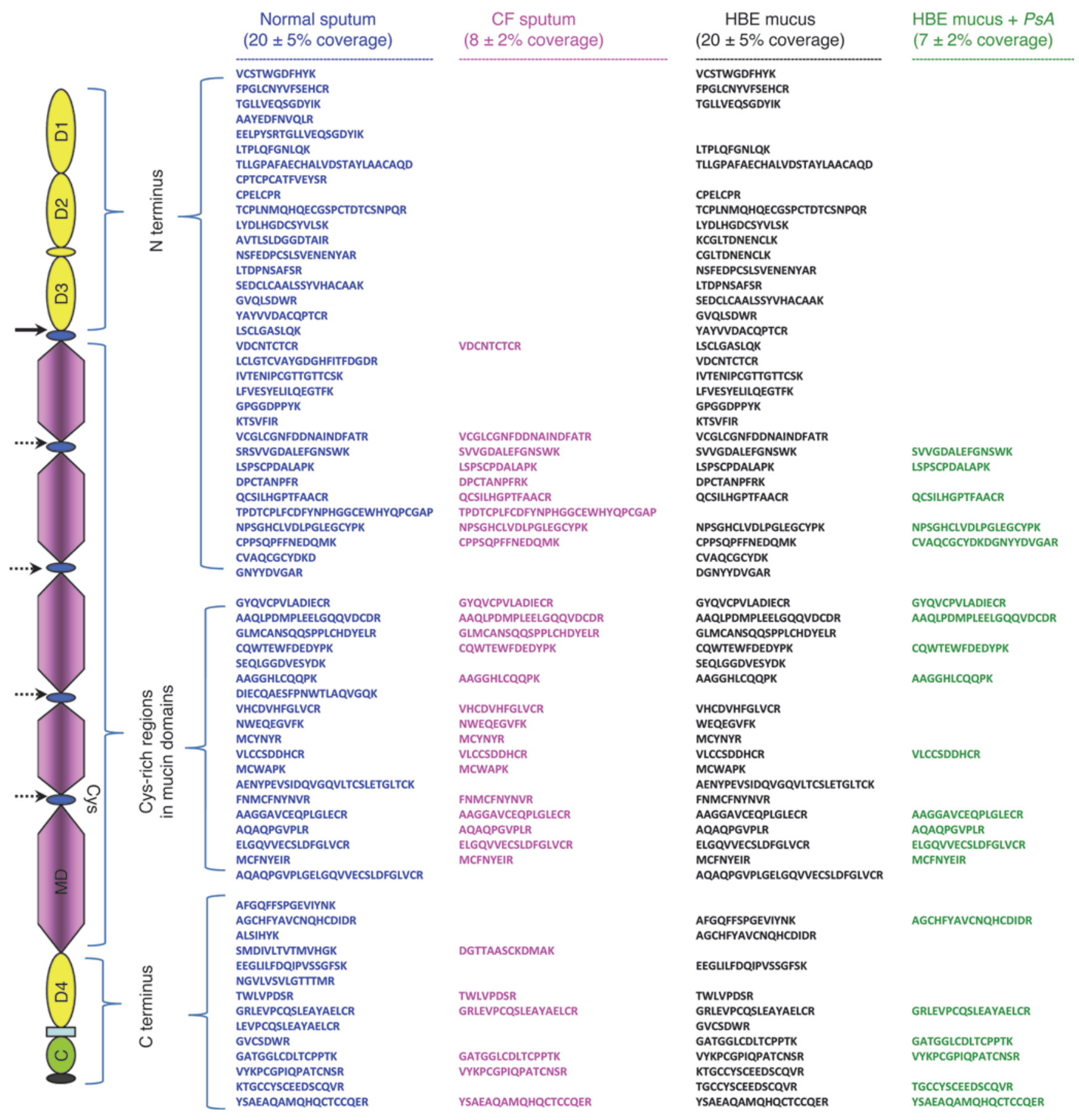

Figure 5

Peptide coverage comparison of MUC5B isolated from normal and CF sputum and HBE secretions with and without $P$. aeruginosa. Mucins were isolated from normal and CF sputum and PaO-treated and nontreated HBE cell culture mucus samples using density gradient centrifugation. Mucins were then digested with trypsin, and peptides were identified by LC-MS/MS analysis. The coverage map indicates that most of the peptides from the $\mathrm{N}$ terminus region, including vWF domains, were not readily detectable in CF sputum and PaO-treated HBE mucus, suggesting that the integrity of these regions was affected by the proteolytic activity. Mucin domains are shown as (von Willebrand) D1, D2, D3, D4, C domains, cystein-rich (Cys), and O-glycosylated tandem repeat mucin domains (MD). Dotted arrows illustrate repeating positions of the epitope (RNREQVGKFKMC) for one of the MUC5B antibodies used (and the antibody used in the study by Henke et al.; ref. 17). The solid arrow illustrates the positions of the epitope (CSWYNGHRPEPGLG) for the other MUC5B antibody used. See Table 1 for antibody details. 
A
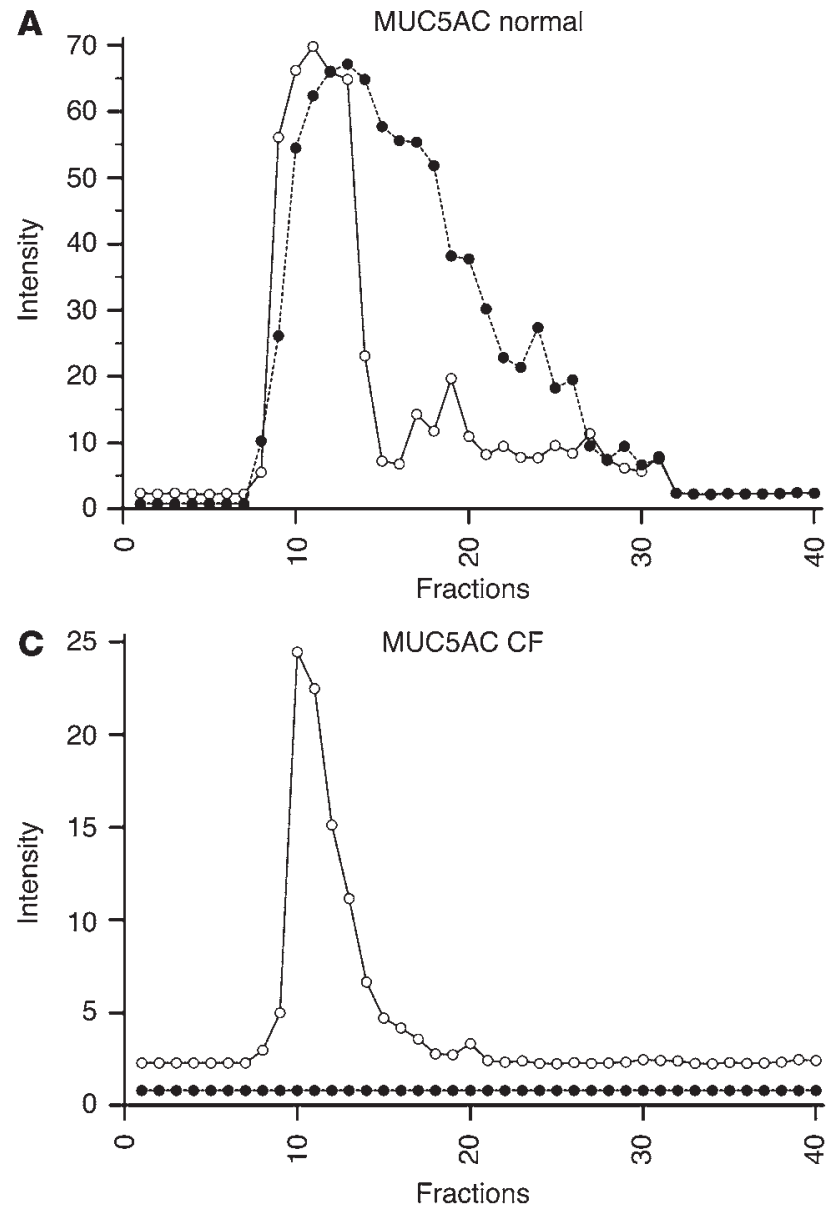
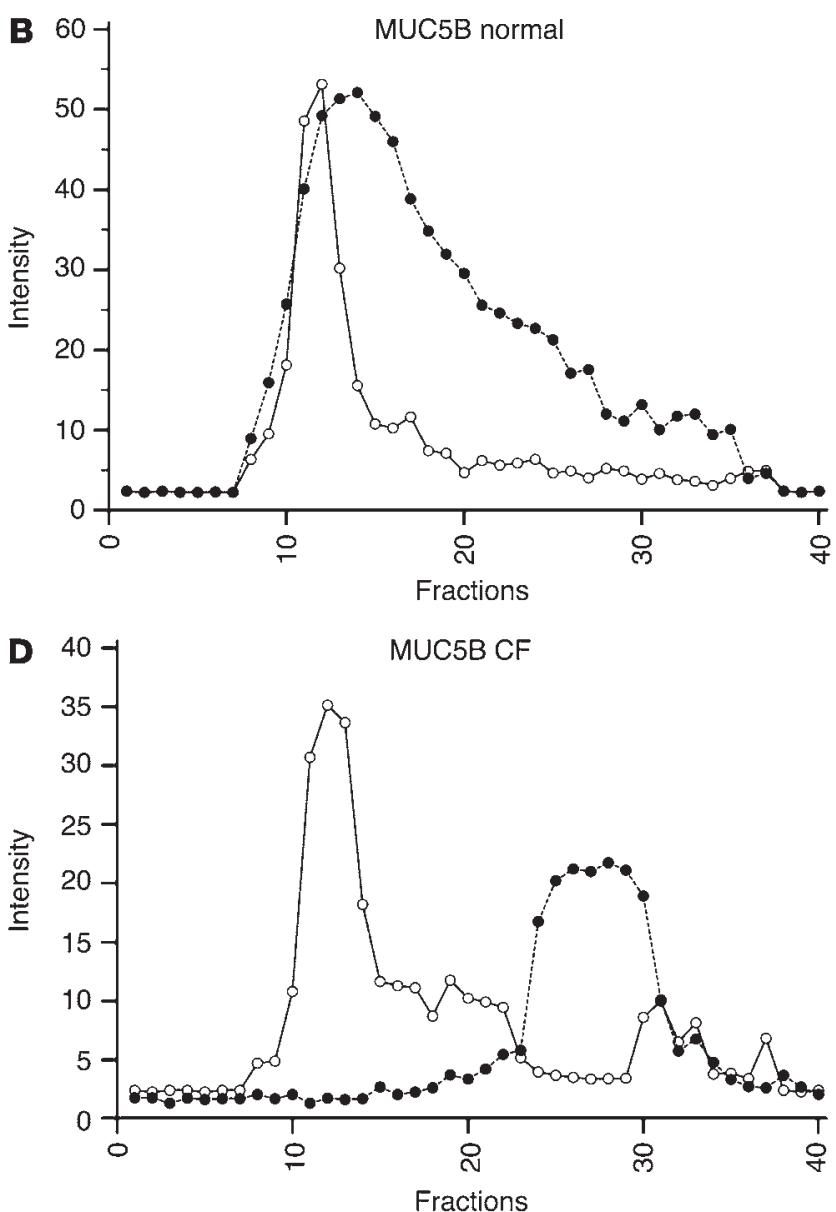

Figure 6

Distribution of mucin macromolecules in normal and CF sputum as assessed by gel filtration chromatography. Sputum samples were subjected to Sepharose CL-2B chromatography. Fractions collected were analyzed via slot blot and probed with MUC5AC (A and C) or MUC5B (B and D) antisera. Solid line with white circles and dotted lines with black circles represent unreduced and reduced samples, respectively. Chromatograms are representative of at least 3 independent experiments.

as described in Methods, and subjected to Sepharose 2B column refractometry-based (SEC/dRI-based) total mucin quantitation analyses (Figure 10A). The total mucin contents of the normal sputum samples were distributed between $1,014 \mu \mathrm{g} / \mathrm{ml}$ and $4,755 \mu \mathrm{g} / \mathrm{ml}$ (average: $2,710 \mu \mathrm{g} / \mathrm{ml}$ ). The mucin concentrations of sputum from CF samples ranged between 3,484 and $8,902 \mu \mathrm{g} / \mathrm{ml}$, with $6,454 \mu \mathrm{g} / \mathrm{ml}$ being the average total mucin concentration. The differences in the values among the 2 groups were statistically significant $(P=0.001)$. Analyses of the efficacy of DNase treatment revealed that virtually $100 \%$ of DNA was removed from the measured sample (see Supplemental Figure 1). Thus, these data suggest that mucin concentrations in whole CF secretions were higher than normal.

\section{Measurements of mucus partial osmotic pressure}

Sputum samples and mucus obtained directly from CF lungs at transplantation were subjected to combined measurements of mucus concentration (percentage of solids, hereafter referred to as \% solids), performed gravimetrically, and of partial osmotic pressures. We found that normal sputum exhibited \% solids values of $1.15 \pm 0.29$ SD and partial osmotic pressures of $75 \pm 24 \mathrm{~Pa} \mathrm{SD}$, both within ranges predicted for normal subjects (ref. 2 and Figure 10B). For reference, the predicted osmotic pressure of the periciliary liquid layer (PCL) is noted by the dashed line at approximately $500 \mathrm{~Pa} \mathrm{(2).}$ In contrast, the CF sputum \% solids values were $5.61 \pm 1.92 \mathrm{SD}$, approximately 5 -fold higher than normal, and the partial osmotic pressures averaged $541 \mathrm{~Pa} \pm 206 \mathrm{~Pa}$ SD. Importantly, the mean partial osmotic pressure increased by approximately 7-fold compared with normal pressure. Finally, mucus retained in the CF lung exhibited \% solids values of $12.12 \pm 1.97 \mathrm{SD}$ and partial osmotic

Table 1

Antibodies used for mucin immunodetection

$\begin{array}{lccc}\text { Target } & \text { Antibody } & \text { Epitope/peptide } & \text { Reference } \\ \text { MUC5AC } & \text { LUM5-1 (polyclonal) } & \text { RNQDQQGPFKMC } & 12 \\ & \text { 45M1 (monoclonal) } & \text { C-terminal cystein-rich part } & 48 \\ \text { MUC5B } & \text { EU-MUC5Ba (monoclonal) } & \text { RNREQVGKFKMC } & 44 \\ & \text { MAN5BIII (polyclonal) } & \text { CSWYNGHRPEPGLG } & 43 \\ & \text { MUC5B222 (polyclonal) } & \text { CQPQCQWTKWIDVDY } & 49\end{array}$




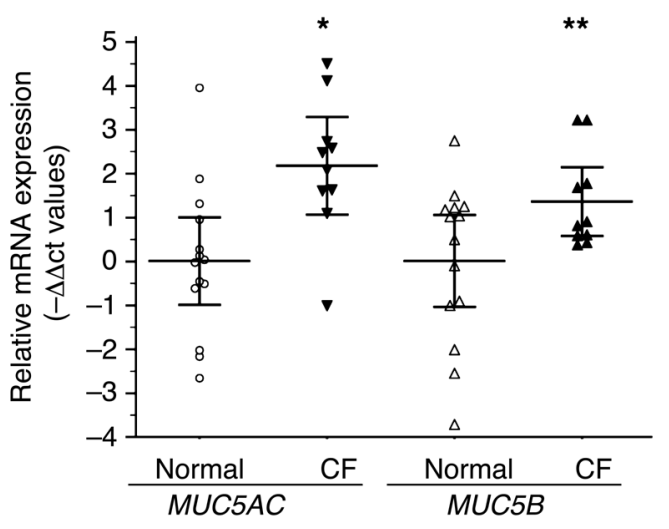

\section{Figure 7}

Changes in mucin mRNA expression in CF lungs as monitored by relative quantitative real-time PCR. mRNA was collected from non-CF $(n=14)$ and CF $(n=11)$ lungs at the time of death or transplantation. Sampling occurred in cartilaginous airways ( $0.8 \mathrm{~mm}$ in diameter) and consisted of epithelial cells devoid of submucosal glands. The comparative Ct $(\Delta \Delta \mathrm{Ct})$ method was used to determine changes in cross-point values for MUC5AC $\left({ }^{\star} P=0.003\right)$ and MUC5B $\left({ }^{\star \star} P=0.024\right)$ using $18 S$. Mean and standard error values are indicated by major (mean) and minor (SEM) horizontal bars. pressures of 2,152 $\mathrm{Pa} \pm 1,118.1 \mathrm{~Pa} \mathrm{SD}$, higher than those found in either CF or normal sputum. Overall, there was a good agreement (correlation coefficient $=0.928$ ) between sample $\%$ solids concentration and mucus partial osmotic pressure over the whole range of samples studied.

To allow comparisons of the relationships between $\%$ solids and partial osmotic pressures and mucin concentrations, we measured both \% solids values and mucin concentrations by SEC/dRI in normal and CF sputum samples. As shown in Supplemental Figure 3, we observed a linear relationship $\left(R^{2}=0.9855\right)$ between \% solids values of mucus and mucin concentrations.

\section{Discussion}

It is critical that mucus be continually cleared from airway surfaces for host defense of the lung. The transportability of mucus is highly dependent on the non-Newtonian biophysical properties of mucus that are governed by the concentration of mucins in the mucus layer (2). As mucin concentrations increase, changes in the viscoelastic properties of the layer emerge, slowing mucus clearance (9). However, the probable key step in the pathogenesis of airway diseases is mucus stasis and mucus adherence to airway surfaces $(31,32)$. Recent evidence suggests that a biophysical parameter that predicts stasis is the partial osmotic pressure of the mucus layer (2). As with viscoelastic properties, the partial osmotic pressure of the mucus layer is a function of the mucin concentration. Therefore, in evaluating the role of abnormal mucus transport in the pathogenesis of CF lung disease, knowledge of the absolute mucin concentration and partial osmotic pressures of $\mathrm{CF}$ and normal airway secretions is required.

Historically, there have been technical difficulties in measuring mucin concentrations even in normal biologic fluids, in large part reflecting the enormous size of mucin macromolecules and the fact that the protein backbone is heavily shielded by $O$-linked sugars. As shown in Figure 5, mucins have "naked" terminal protein regions that are important for dimerization, higher-order multimerization, and protein-protein interactions. Typically, it is these exposed regions in the $\mathrm{N}$ and $\mathrm{C}$ termini and the Cys region of MUC5AC and MUC5B against which antibodies have been generated for immunologic quantitation of mucins. A problem is that these regions are also the regions exposed to endogenous $(17,33)$ and exogenous $(25)$ proteolytic activities, which have been reported to cleave these regions of mucin macromolecules $(18,25)$. Thus, these considerations suggest that there may be major technical difficulties measuring mucin concentrations by immunologic techniques in biologic solutions containing active proteases.
Like Henke et al. (17), our immunologically based quantitative analyses of mucins revealed decreased levels of whole and reduced MUC5B in adult $P$. aeruginosa-infected CF sputum compared with levels in normal sputum (Figure 1). Our data from BAL samples from pediatric control versus pediatric CF patients revealed the predicted increase in concentrations of the globular protein lactoferrin in CF but, like Henke et al. reported, decreased concentrations of MUC5B (Figure 2). Because of the reported presence of NE in CF airway secretions early in life (34), we directly tested the hypothesis that human NE (HNE) could proteolytically attack the "naked" protein regions of MUC5AC and 5B, the regions against which our antibodies were directed. As shown in Figure 3, we observed time-dependent decreases in immunoreactive MUC5B following exposure to HNE or trypsin. Importantly, there was relatively little change in the molecular mass of mucins following this exposure. These findings raised the possibility that elastase cleavages affect sites in mucin monomers not critical for oligomeric (macromolecular) behavior (see below).

We also investigated the possibility that bacterial proteases contribute to proteolysis of antigenically exposed sites of MUC5B. These studies revealed that $P$. aeruginosa, growing in thick mucus with a biofilm physiology, produced an elastase-like activity that cleaved mucin macromolecules and reduced their immunologically measured concentrations (Figure 4). PAK bacteria genetically deficient in the capacity to produce biofilms and secrete elastase did not exhibit this activity. In contrast to the data for sputumor saliva-exposed HNE, $P$. aeruginosa-secreted proteases produced a greater reduction in mucin molecular mass, suggesting that $P$. aeruginosa proteases may not be the dominant mucin-cleaving proteases in vivo (compare Figure 4 with Figure 1).

The mass spectrometric analyses comparing the mucins in CF versus normal sputum revealed key data that accounted for this apparent reduction in mucin concentrations in CF sputum as measured immunologically. The mass spectrometric studies produced evidence for mucin proteolysis centered on the "naked" $\mathrm{N}$ and $\mathrm{C}$ termini, which was revealed by the absence of peptide coverage in these regions (Figure 5). Similar mass spectrometric analyses of sterile HBE culture secretions (control) versus HBE mucus samples that were $P$. aeruginosa exposed revealed a virtually total absence of peptide coverage from the MUC5B N terminus and a significant reduction in peptide coverage from Cys-rich and $\mathrm{C}$ terminus regions in the PAK-exposed samples compared with control HBE samples. These are the regions against which most of the MUC5B antibodies were directed, including those used by 
A
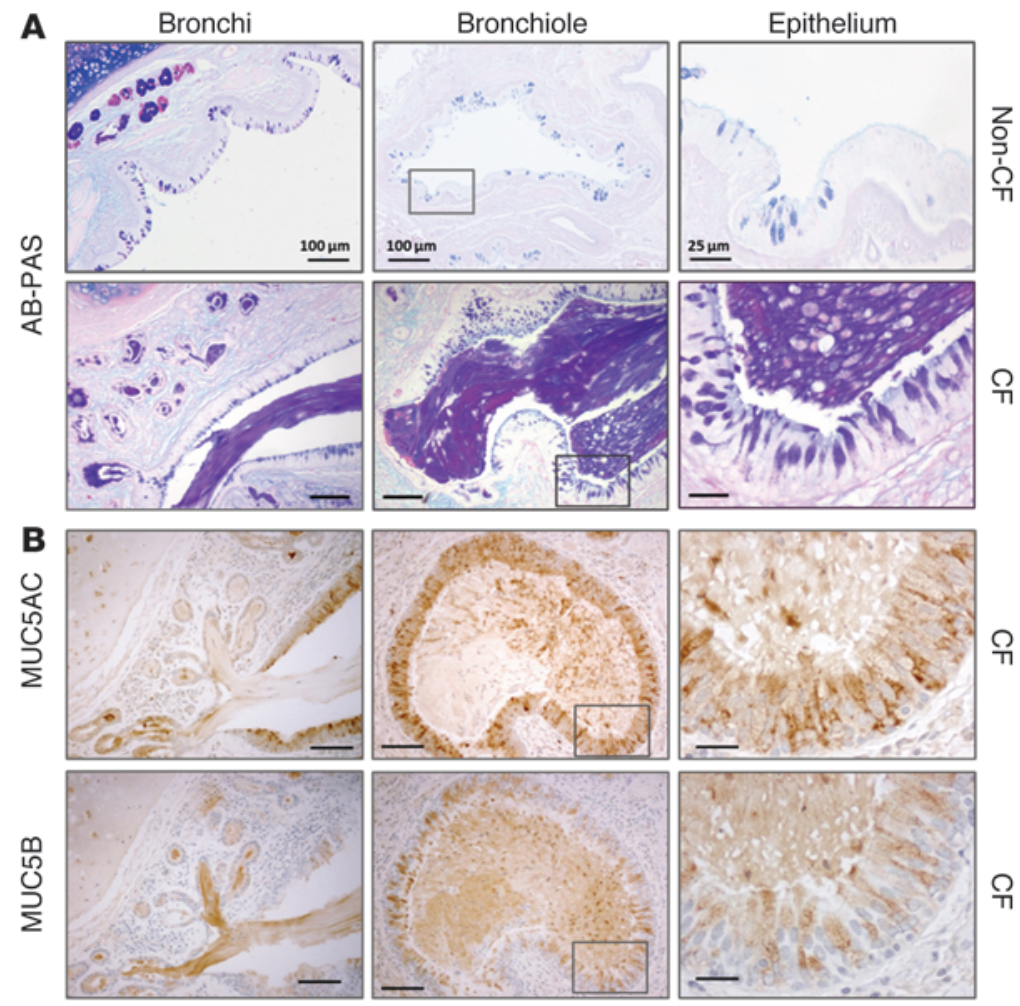

\section{Figure 8}

Histopathological analysis demonstrates the presence of mucus plugs and intracellular mucins in CF lungs. (A) AB-PAS staining of proximal (bronchi) and distal (bronchiole) airways from non-CF and CF lungs show increased AB-PAS-positive material in CF airway surfaces and lumen. (B) MUC5AC and MUC5B immunolabeling of consecutive CF sections revealed that both mucins were expressed by airway goblet cells and participated in mucus plugs. Scale bars: $100 \mu \mathrm{m}$ (Bronchi and Bronchiole columns); 25 um (Epithelium column).

dominant elution of MUC5B and MUC5AC from nonreduced $C F$ samples in the $V_{0}$ fraction suggests that underestimation was not a major problem (Figure 6). Our approach to the DNA overestimation problem was to cleave all DNA with exogenous DNase prior to running the sample on the column. Our internal controls suggest that DNase treatment was effective in removing DNA from the $V_{o}$ volume prior to densitometric quantitation, indicating that there was not a DNA-dependent overestimation of mucin concentrations (Supplemental Figure 1).

We also used an HBE model system to compare immunologic versus physical measurements of mucin concentration in control (PBS) versus proteolytic (fractionated SMM) environments (Figure 9). The physical SEC/dRI approach detected equivalent mucin concentrations in both fractionated SMM and PBS environ-

Henke et al. (17) and ourselves. Thus, these data suggest that there will be a general problem with respect to antibody-dependent measurement of mucins in CF secretions in which free proteases can cleave the antigenically exposed regions of mucins.

The column chromatographic analyses of mucins from normal whole, i.e., nonreduced, samples revealed the expected high molecular weight forms for MUC5AC and MUC5B (Figure 6, $A$ and B). Importantly, we found that the high molecular weight oligomeric structure was preserved in MUC5AC and MUC5B from nonreduced CF samples (Figure 6, C and D). However, in CF samples, we observed a dramatic reduction in MUC5B size and a complete loss of antigenicity of MUC5AC under reducing conditions. These data suggest that despite mucin intramolecular proteolytic cleavage, mucins in CF secretions under physiologic, nonreducing conditions maintain their oligomeric structure by intra- and intermolecular disulfide (S-S) bonds (Figure 11). Exposure of HBE mucus samples to HNE did not reduce sheer dependent viscosity, consistent with the presence of oligomeric mucin structures after proteolytic cleavage (Supplemental Figure 2). Hence, intramolecular cleavage may not affect the important biophysical properties of mucins that determine key viscoelastic and osmotic properties of mucus.

Physical and biophysical methods have been developed to measure mucin concentrations by antibody-independent approaches (35). These approaches use size-selective gel filtration techniques to isolate high molecular weight polymers in $\mathrm{V}_{\mathrm{o}}$ volumes, followed by analysis of the molecular size and concentration of mucins by light scattering and refractometry, respectively. These approaches can underestimate mucin concentrations in proteolyzed sputum. In contrast, the concentration of mucins can be overestimated by the coelution of other large molecular weight polymers, e.g., DNA, in the $V_{o}$, as may be a problem for CF secretion quantitation. The pre- ments, whereas values measured by immunologic techniques in parallel samples were reduced in fractionated SMM versus PBS samples, consistent with SMM-mediated proteolytic cleavage of mucin epitopes. Therefore, we posited that data from SEC/dRI would yield more accurate measurements of mucin concentrations in CF sputum than in normal sputum.

Using biophysical measurement techniques, CF airway secretions contained approximately 3 -fold higher concentrations of secreted mucins than did normal airway secretions (Figure 10A). Note that the normal samples were obtained by sputum induction, as required for sputum sampling in this subject population. Previous unpublished data in chronic obstructive pulmonary disease (COPD) subjects have suggested that sputum induction may underestimate spontaneous mucus weight percentages (wt $\%$ ) by approximately $25 \%$. Conversely, CF lung disease is heterogeneous, with diseased areas admixed with apparently normal areas. Further, CF patients have areas of their lungs in which mucus is so adherent that it cannot be cleared by coughing and, hence, are underrepresented in a sputum sample (see Figure 10B, below). Thus, wt $\%$ of CF secretions could be underestimated by this heterogeneity. On balance, it appears likely that diseased areas in the CF lung are characterized by mucin hyperconcentration.

However, it was important to test whether CF mucins remained sufficiently intact to exert the predicted pathophysiologic defects from increased concentrations. In a new formulation describing the biophysical basis of mucus transport, the transportability of the mucus layer in health requires that the partial osmotic pressure of the mucins in the mucus layer be lower than the partial osmotic pressure of the high molecular weight polymers in a newly defined periciliary gel layer (2). In 

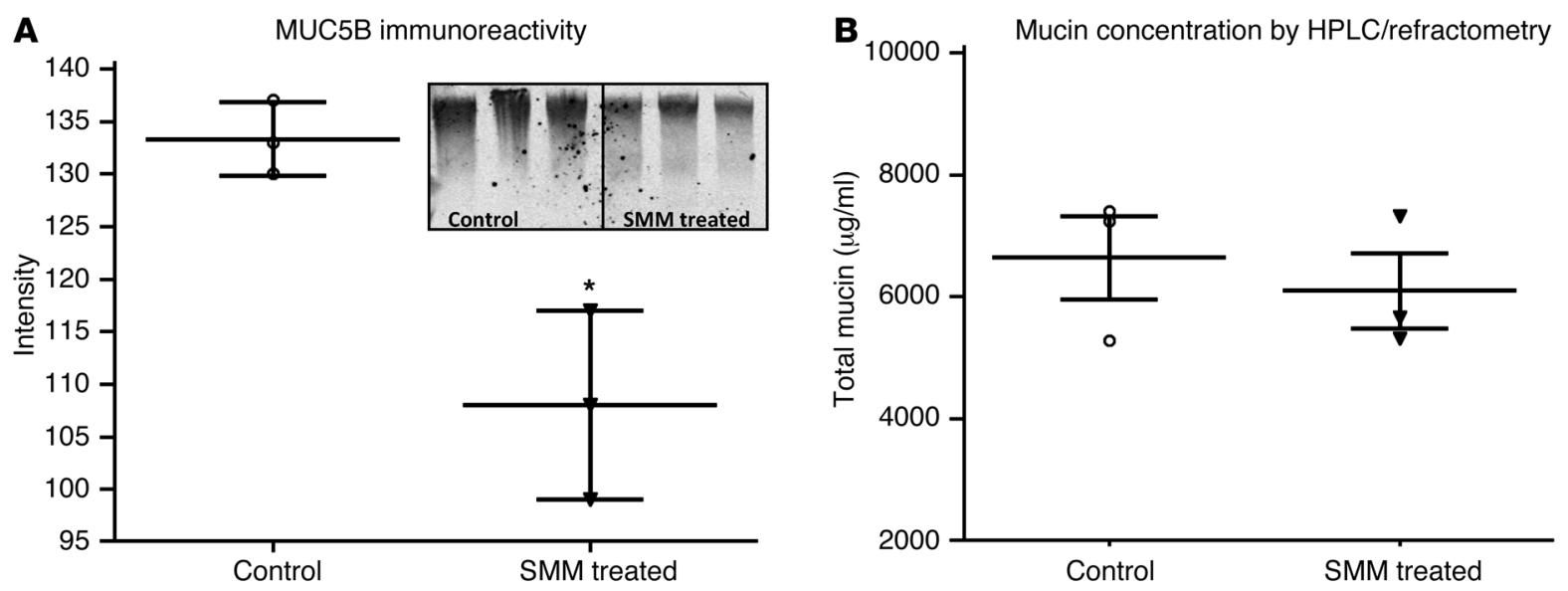

\section{Figure 9}

The effect of CF SMM on mucin immunoreactivity and concentration. HBE cultures, preconditioned by a 72-hour luminal incubation with SMM, were stimulated to secrete mucins and formed mucus with ATP $\gamma$ S. Cultures were then incubated in situ with PBS or with fractionated SMM (100-kDa cutoff) and analyzed for MUC5B by agarose Western blotting (A) or SEC/dRI (B). Note the apparent decrease in MUC5B content in the sample incubated with fractionated SMM, as measured by immunoreactivity $\left({ }^{*} P=0.01 ; n=6\right)$ and the lack of a difference by refractometry measurements $(P>0.5)$. Mean and SEM values are indicated by major and minor horizontal bars, respectively.

vitro data have suggested that the periciliary gel is composed of tethered mucins and other high molecular weight glycoconjugates that are, surprisingly, denser than the secreted mucins in the normal mucus layer $(2,36)$. This notion is consistent with the observation that the partial osmotic pressure of the periciliary layer is approximately $500 \mathrm{~Pa}$ as compared with approximately $100 \mathrm{~Pa}$ predicted for a normal mucus layer. This new paradigm predicts that as the hydration of the airway surface decreases, as with CF, water preferentially leaves the mucus layer, and its partial osmotic pressure rises. When the osmotic pressure of the mucus layer approximates that of the PCL, the mucus layer begins to compress the PCL, and ciliary beating and mucus transport become unstable. At levels at which the partial osmotic pressure of the mucus layer greatly exceeds that of the PCL, cilia collapse, and the mucus layer is compressed onto the CF airway surfaces and becomes adherent, even in response to coughing. Thus, a key question with respect to our studies was whether the mucins in the CF lung with an actively proteolytic environment maintained their osmotic properties.

A technique to measure osmotic pressures of the mucins (partial osmotic pressures) had been developed and was modified for measuring partial osmotic pressures in sputum samples (2). As shown in Figure 10B, the partial osmotic pressure of induced sputum from normal subjects was approximately $100 \mathrm{~Pa}$, closely approximating the values suggested by modeling studies and studies of normal HBE cultures in vitro. The partial osmotic pressure of the sputum obtained from CF subjects was in the 500-Pa range, close to the partial osmotic pressure of the PCL. This is precisely within the range that might be predicted for mucus to be poorly cleared and moderately adherent, but able to be cleared by coughing. Importantly, CF airway secretions that could not be cleared by coughing, i.e., those obtained from direct removal of secretions from CF lungs excised at transplantation, exhibited very high partial osmotic pressures, i.e., pressures greater than 1,000 $\mathrm{Pa}$. Note that the partial osmotic pressures were a direct function of the mucus \% solids, an accurate index of mucin concentration, as shown in Supplemental Figure 3.
Thus, these data suggest that CF secretions exhibit the osmotic pressures predicted by mucin concentrations, i.e., mucin polymer osmotic properties and are not grossly affected by proteolysis. The analysis of the ability of CF mucus to be cleared based on $\%$ solids and/or osmotic pressure may serve as a useful biomarker for novel therapies to treat CF lung disease.

Based on these data, we hypothesize that the following scenario may occur in the CF airway. Initially, CF mucins are likely heterogeneously concentrated by the volume depletion that results from absent CFTR function and an exogenous stress, e.g., viral infection or aspiration (37). Concentrated mucins produce mucus adhesion to airway surfaces with resultant bacterial infection and inflammation. With persistent bacterial infection and consequent inflammation, CF airway epithelia remodel to exhibit goblet cell hyperplasia and consequent increased mucin gene transcription. This latter notion is consistent with the measured elevation in mucin mRNA for MUC5AC and MUC5B, as shown in Figure 7. Mucin RNA transcripts are translated into MUC5AC and MUC5B proteins that are $O$-glycosylated and stored. Although there have been reports of an absence of goblet cell hyperplasia in CF (38), our data (Figure 8) and those of others (15) would suggest that the increase in MUC5B and MUC5AC in RNA is associated with goblet cell hyperplasia. The secreted MUC5AC and MUC5B proteins are attacked in the lumen by proteolytic enzymes released by inflammatory cells and bacteria, and the naked epitopes are degraded, but the macromolecules are held in place by the persistence of disulfide bonds between intra- and intermolecular cysteines. This latter notion rests on the relative absence of a change in size versus a large loss of immunoreactivity when comparing unreduced and reduced CF samples by Sepharose chromatography (Figure 6) and on the maintenance of molecular mass following elastase exposure (Figure 3B). These data would therefore suggest that non-Newtonian properties will be maintained by intact mucin macromolecules in infected CF mucus (Supplemental Figure 2). Thus, the increased CF mucin concentrations will exert increased osmotic effects (Figure 10B) and abnormal viscoelastic properties (39) at both the early preinfected and the later mucoinfected stages of CF. 


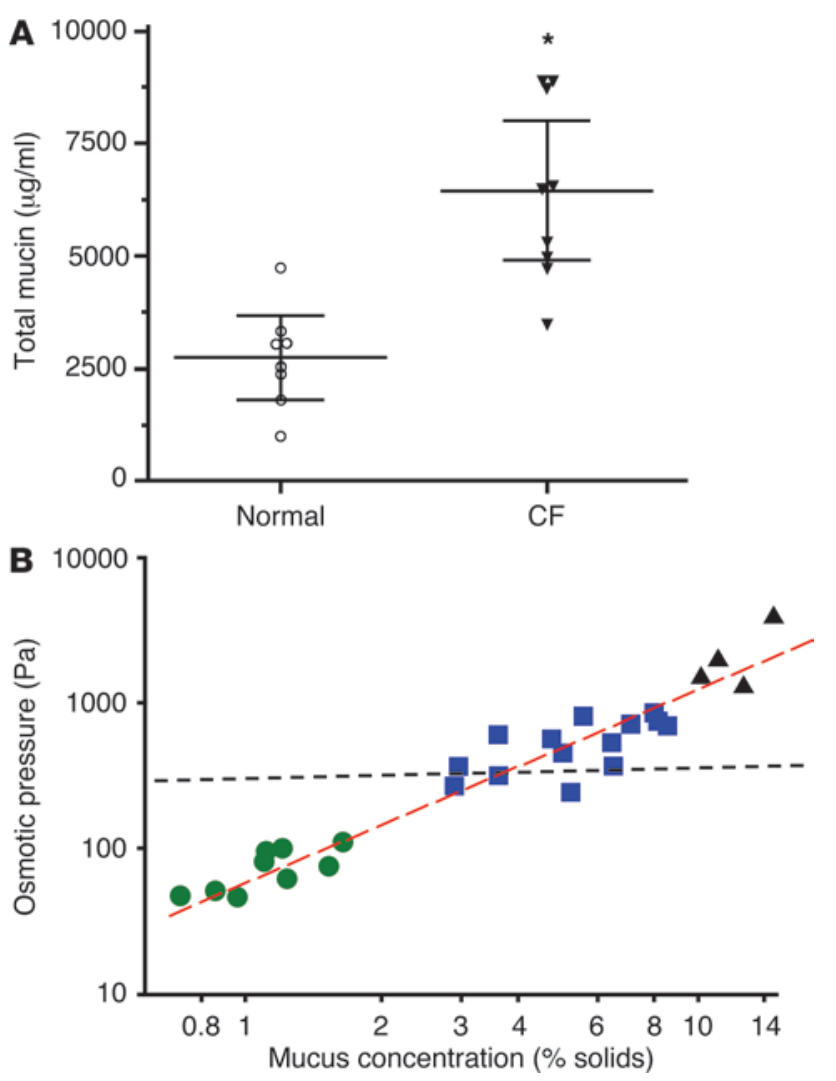

Figure 10

Changes in total mucin concentrations in CF sputum and relationship between mucus concentration and osmotic pressure. (A) Total mucin concentrations of sputum samples from normal and CF lungs were measured by SEC/dRI. Calculated concentrations of each sample were plotted for comparison. Mean and SEM values are indicated by major and minor horizontal bars, respectively. An independent samples $t$ test was used to determine changes in values between normal and CF samples, indicating a significant increase in total mucin concentration in CF samples ( $\left.{ }^{*} P=0.001\right)$. (B) Relationship between the osmotic pressure of human-derived mucus samples (measured with a $10-\mathrm{kDa}$ membrane) and their total mucus concentration (i.e., \% solids). Samples depicted were from normal (green circles) and CF (blue squares) sputum and undiluted airway mucus samples obtained from excised CF lungs at the time of transplantation (black triangles). Red dashed line represents the best fit with a log-log correlation coefficient of 0.928 . Black horizontal dashed line represents the predicted osmotic pressure of the PCL. Note that mucus osmotic pressures above the black dashed line are predicted to produce PCL collapse.

In summary, mucin concentrations in CF secretions from young adults are approximately 3 times higher than normal controls as measured by physical techniques. Immunologic techniques are likely not suitable for measuring mucin concentrations in airway environments characterized by free proteolytic activities. Despite proteolysis, CF mucins appear to retain their polymeric structure and biophysical properties, in particular, their increased osmotic activity ( $\sim 5$ times), which may be a key biophysical step in achieving mucus stasis. These findings point to the need to measure the mucin concentrations in the heterogeneously affected diseased area early in the pathogenesis of CF lung disease, i.e., in neonates, to definitively identify the role of excessively concentrated mucus in initiating CF lung disease. The recent reports on free HNE and BAL of CF neonates suggest that biophysical approaches will be required for these measurements (34). Finally, these data suggest that logical therapies for CF lung disease would include means to dilute the mucins in CF secretions so their osmotic pressures fall below those of the PCL and/ or reduce the rates of mucin secretion.

\section{Methods}

\section{Collection of sputum and BAL}

Samples were collected as detailed in the Supplemental Methods. Briefly, spontaneous sputum was collected from anonymized CF subjects or consented CF subjects with $P$. aeruginosa infection. In addition, induced sputum was collected from consented normal volunteers as previously described (35). The demographic data for each patient group are presented in Supplemental Table 1. Pediatric BAL samples were obtained during bronchoscopy for clinical indications using standard methods of collection (ref. 40 and Supplemental Methods).

Freshly excised normal and CF lungs for immunohistochemical and molecular measurements of mucin mRNA expression and mucin secretory cell numbers. Fourteen non-CF and 10 CF lungs were analyzed by real-time PCR for MUC5B and MUC5AC mRNA expression and immunochemical localization of MUC5AC and MUC5B (Figure 7 and Figure 8). Non-CF lung specimens were collected postmortem from head trauma victims after 1 to 3 days of mechanical ventilation. All CF specimens were collected at lung transplantation without prior mechanical ventilation. Organ harvest and acceptability criteria have previously been described in detail (41).

\section{HBE cultures}

Cartilaginous bronchi were obtained from normal lungs not eligible for transplantation. Cells were isolated by protease digestion, expanded on tissue culture plastic in bronchial epithelial growth medium, and then plated on an air-liquid interface (ALI) system with ALI media as previously described (41). The cultures were used both as a source of mucus and for the mucin secretion measurements (see below).

\section{Immunological and physical measurements of mucin properties in sputum and cell culture samples}

Reduction of samples. Samples were reduced in $10 \mathrm{mM} \mathrm{DTT}$ at $37^{\circ} \mathrm{C}$ for 2 hours. Iodoacetomide $(20 \mathrm{mM})$ was then added to samples for carboxymethylation at room temperature, in the dark for 30 minutes. The samples were then analyzed by slot blot or agarose gel electrophoresis (see below). For descriptive purposes, these samples will subsequently be referred to only as "reduced," although they were also carboxymethylated.

Gel electrophoresis and immunoblotting. Pediatric BAL fluid, sputum, and cell culture mucus samples were loaded at a constant volume (usually $30 \mu \mathrm{l}$, undiluted) onto a $0.7 \%$ agarose gel in a $1 \mathrm{X}$ Tris-acetate-EDTA (TAE) buffer with $1 \%$ SDS and electrophoresed in a horizontal gel apparatus as previously described (42). After electrophoresis, the gel was reduced in $10 \mathrm{mM}$ DTT for 20 minutes prior to vacuum transfer onto a nitrocellulose membrane. The proteins were transferred by vacuum transfer in $4 \times$ saline-sodium citrate (SSC) buffer at 45 mbar for 1.5 hours. Membranes were then probed for mucins using specific antibodies raised against MUC5AC (12) and MUC5B (43).

\section{MUC5B purification and incubation with proteases}

MUC5B was isolated from saliva, collected anonymously from normal volunteers (IRB exempted), as previously described (24). Purified MUC5B $(100 \mu \mathrm{g} / \mathrm{ml})$ was dialyzed against PBS, preheated to $37^{\circ} \mathrm{C}$, and 


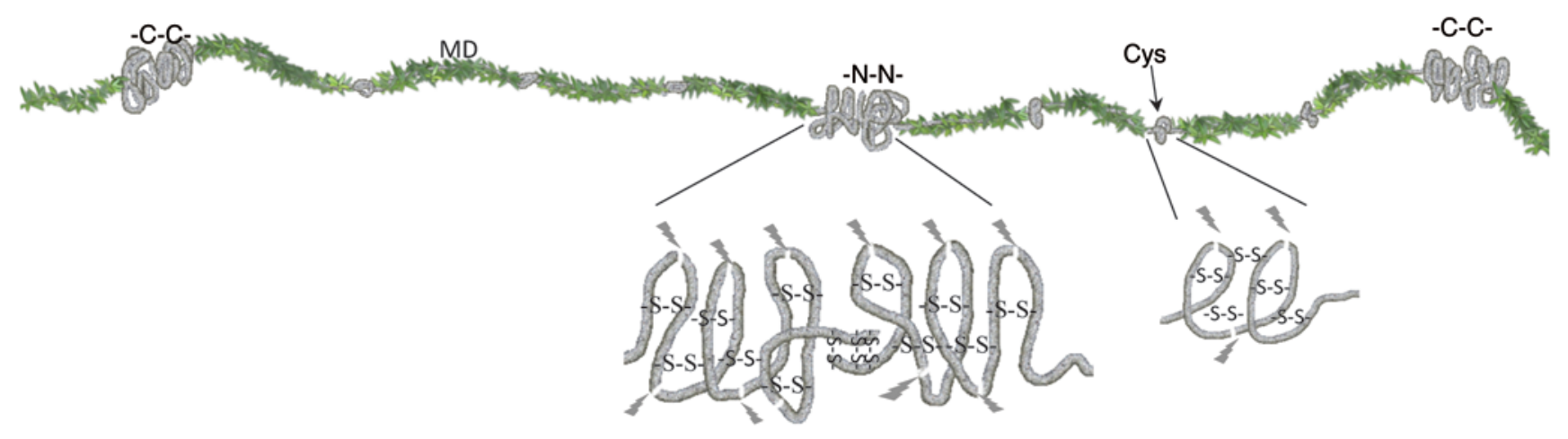

\section{Figure 11}

Proposed effect of proteolytic cleavages on mucin integrity. Two mucin monomers are depicted, with glycosylated tandem repeat mucin domains depicted in green and "naked" Cys domains and N and C termini depicted in gray. Enlargements (below) show naked N-and C-terminal protein regions, where the molecules that are susceptible to proteolysis (highlighted by lightning bolts) oligomerize (C- to $\mathrm{C}$ - and $\mathrm{N}$ - to $\mathrm{N}$-) and cystein-rich regions that are also protease targets. Depending on the cleavage site(s), antibody immunoreactivity will be affected by these cleavages. Conversely, macromolecular integrity of the mucins are not immediately affected by cleavage, since the oligomers are held together by disulfide bonds.

incubated with proteases, $1 \mu \mathrm{g} / \mathrm{ml}$ trypsin (Sigma-Aldrich, modified from porcine pancreas), and $1 \mu \mathrm{g} / \mathrm{ml}$ human elastase from human neutrophils (MP Biomedicals) for 5, 20, 60, and 240 minutes. SDS-PAGE running buffer (in $8 \mathrm{M}$ urea) was added to the samples to stop the reaction at each time point. An aliquot from the starting sample treated with PBS served as a control. Samples were then subjected to agarose gel electrophoresis and immunoblotted with 2 different MUC5B antibodies, including a monoclonal antibody (44) and polyclonal antisera (43). Also, an aliquot from samples was subjected to SEC/MALS/dRI analyses. Samples were chromatographed on a Sepharose CL-2B $(2 \times 5 \mathrm{ml})$ column and eluted with PBS at a flow rate of $400 \mu \mathrm{l} / \mathrm{minute}$. The column effluent was passed through an online Dawn (Wyatt Technology) MALS instrument coupled to a Wyatt Optilab inferometric refractometer to measure molecular mass and mucin concentration, respectively. The captured data were integrated and analyzed with the Astra software provided with the Dawn instrument.

\section{"Normal" versus concentrated mucus harvested from HBE cell cultures with and without exposure to $P$. aeruginosa}

Mucus was harvested from $\mathrm{HBE}$ cell cultures and concentrated to $2.5 \%$ solids (i.e., "normal" concentration) or $8 \%$ solids ("CF-like" concentration) as previously described (26). These 2 different concentrations of HBE mucus were incubated for 72 hours with a lab strain of $P$. aeruginosa $\left(\mathrm{PAO} 1,5 \times 10^{3}\right.$ $\mathrm{CFU}$ ) or a mutant form of $P$. aeruginosa (PAO-JPI, $5 \times 10^{3} \mathrm{CFU}$ ) lacking the lasI quorum-sensing gene, which controls $P$. aeruginosa elastase gene expression and therefore expresses minimal elastase activity (27). Mucus was then harvested at 72 hours for mucin analyses by agarose Western blotting and for peptide coverage by mass spectrometry.

\section{Measurement of lactoferrin}

The lactoferrin content of pediatric BAL fluids was measured by a sandwich ELISA using a lactoferrin IgG for capture (Accurate Chemical) and lactoferrin IgG-HRP for detection (Cappel, MP Biomedicals). After coating with lactoferrin IgG, the microtiter plates were washed and blocked with $2 \%$ BSA and $0.1 \%$ Tween- 20 in PBS, and $90 \mu \mathrm{l}$ of BAL fluid was added to each well for a 30 -minute incubation at room temperature. The plates were washed with PBST and developed with o-phenylenediamine dihydrochloride. Standards, consisting of commercial human lactoferrin (L4040; Sigma-Aldrich) were applied to the same plates as the samples.

\section{Mass spectrometry of mucins to determine protein integrity}

Mass spectrometric analyses of purified mucins were performed as described previously (35). Mucins were first purified from sputum or cell culture mucus samples by isopycnic density gradient centrifugation with a starting density of $1.45 \mathrm{~g} / \mathrm{ml}$. Samples were subjected to ultracentrifugation at $145,000 \mathrm{~g}$ for approximately 65 hours. The samples were then unloaded into $2-\mathrm{ml}$ fractions (total of 20 fractions) and analyzed via slot blot and gel electrophoresis. PAS-rich mucin fractions were pooled, reduced, alkylated, and subjected to trypsin digestion. Tryptic peptides were subjected to liquid chromatography-tandem mass spectrometry (LC-MS/MS) in a Waters Micro-Q mass spectrometer for mucin coverage $(33,35,45)$.

\section{Column chromatography for characterization of mucin oligomeric state in whole versus reduced conditions}

Unreduced and reduced sputum or cell culture samples were diluted and fractionated on a Sepharose CL-2B column. Fractions were collected and subjected to PAS and mucin immunoblotting by slot blot analyses as described previously (46).

\section{Measurement of mucin concentrations using SEC/dRI}

Sputum samples were diluted 1:5 in PBS containing DNase (10 units) and $\mathrm{CaCl}_{2}(1 \mathrm{mM})$ and incubated for 15 minutes at room temperature. Samples were then mixed $1: 1$ with $6 \mathrm{M} \mathrm{GuHCl}$ before analysis. In brief, an aliquot $(100 \mu \mathrm{l})$ of the diluted sputum samples was chromatographed on a Sepharose CL-2B size exclusion column $(15 \times 2.5 \mathrm{~cm})$ and eluted with $0.2 \mathrm{M} \mathrm{NaCl}$ (with $10 \mathrm{mM}$ EDTA) at a flow rate of $500 \mu \mathrm{l} /$ minute. The column effluent was passed through an in-line enhanced optimal system laser photometer (Dawn; Wyatt Technology) coupled to a digital signal-processing inferometric refractometer (Optilab; Wyatt Technology) to continuously measure light scattering and sample concentrations, respectively. Note that molecular size, as measured by light scattering, was used to identify and define the mucin peak. The captured data were integrated and analyzed with the Astra software provided with the Dawn laser photometer. Optimum dilutions were sought by trial and error, and then paired values were obtained to check reproducibility, which was within $5 \%$ on average. Absolute values of mucin concentration were determined from the differential refractometry by measuring the specific refractive index increment called $\mathrm{d} n / \mathrm{d} c$ that reflects the deviation of the refractive index by concentration. A d $n / \mathrm{d} c$ of $0.165 \mathrm{ml} / \mathrm{g}$ was used for mucins, which was used previously for the same (47). 


\section{Measurements of airway mucins MUC5AC and MUC5B in mRNA and mucin secretory cell numbers}

Mucin $m R N A$ by quantitative PCR. Total mRNA was extracted from $\mathrm{CF}$ $(n=10)$ and non-CF $(n=14)$ cartilaginous mainstem bronchi $(\sim 0.8 \mathrm{~cm}$ in diameter). Cross sections of mainstem bronchi were carefully dissected free and opened longitudinally. Surface cells were gently scraped (excluding submucosal glands as verified by histology), placed in RNAlater (Ambion), and processed for mRNA extraction according to the manufacturer's instructions. Total mRNA quality was determined using an mRNA bioanalyzer (Agilent Technologies). The following primers were used for MUC5AC: 5'-AGCTACCCTGCTCCTGGAAT-3' and 5'-GTGGGAGCTGGTTCTTGTTC-3'. The following primers were used for MUC5B: 5'-CTGGCACCTGCTCTGTGCA-3' and 5'-CACTGCTTTGAGGCAGTTCT- $3^{\prime}$. Analysis of mRNA amplification was conducted via a LightCycler 480 II (Roche Diagnostics) reading SYBR green signal.

Immunocytochemical analysis of lung tissues. Cross sections of mainstem bronchi $(\sim 0.8 \mathrm{~cm})$ and bronchioles $(<0.5 \mathrm{~mm})$ were fixed and processed for histologic analyses. Consecutive sections of these specimens were stained by AB-PAS or immunolabeled with an anti-MUC5AC (48) (45M1; Santa Cruz Biotechnology Inc.) or an anti-MUC5B (MUC5B222) antibody (49). Signal was revealed via $\mathrm{DAB}$ and detected via a transmitted light microscope (Nikon Microphot) and captured with a Nikon DXM 1200 camera.

\section{Direct comparison measurements of mucin concentration by immunologic and biophysical techniques following mucin secretion into an SMM from excised CF lungs}

SMM, a gift from Carla Ribeiro (UNC CF Center, Chapel Hill, North Carolina, USA), was generated by aspirating the intraluminal mucopurulent material from freshly excised CF lungs at the time of transplantation (30). The harvested material was then ultracentrifuged and filtered through $0.45-\mu \mathrm{m}$ membranes to remove bacteria. This "neat" SMM was used in 72-hour HBE exposures. A second aliquot of SMM was further fractionated through "fractioned" $100 \mathrm{~K}$ cutoff membranes to remove the endogenous mucins and other high molecular weight substances that would add background to measurements. HBE cultures grown at an ALI were treated with SMM for 72 hours to induce inflammation and mucin secretory cell hyperplasia (30). The SMM-treated cultures were then washed free of SMM with PBS and challenged for 1 hour with a mucin secretagogue, ATP $\gamma \mathrm{S}(100 \mu \mathrm{M})$, in a small volume of PBS $(60 \mu \mathrm{l})$. After this 1-hour incubation, the cultures were exposed to an additional $30 \mu \mathrm{l}$ PBS (control) or $30 \mu \mathrm{l}$ of fractionated SMM, incubated for 2 hours, and the apical surface was then sampled and aliquots reserved for mucus quantitation by Western blotting and SEC/dRI.

\section{Measurement of mucus solids content, osmotic pressure, mucin concentrations, and viscosity}

The percentate of solid content of mucus, an index of hydration, was calculated by measuring wet/dry weights with a microbalance (UMX2; Mettler Toledo) (2). The osmotic pressures of normal sputum, CF sputum, and secretions from excised CF lung samples were measured with modifications of previously described techniques (2). Briefly, a 300- $\mu$ l aliquot of each sample was gently placed on the membrane surface of a custom- designed direct-membrane osmometer. For these studies, a $25-\mathrm{mm}$ diameter, $10-\mathrm{kDa}$ molecular weight cutoff polyethersulfone membrane (Millipore) separated the test chamber from the reference chamber that was filled with PBS. This membrane allowed salt and small peptides and proteins to freely permeate, while retaining larger proteins, including mucins. To permit direct comparisons of mucus \% solids and mucin concentrations, both measurements were made in a series of sputum samples collected from normal and CF subjects. The complex viscosity of the NE-treated samples (Supplemental Figure 2) was determined by shear dependence of the viscosity on a rheometer as detailed in the Supplemental Methods.

\section{Reagents used for the experiments}

Reagents used for the experiments include the following: DTT, sodium metabisulfite solution ( $0.4 \mathrm{~g}$ metabisulfite per $400 \mathrm{ml}$ water with $4 \mathrm{ml} \mathrm{HCl}$ ), periodic acid solution, $10 \times$ TBST ( $12.1 \mathrm{~g}$ Tris $\mathrm{HCl}, 87.6 \mathrm{~g} \mathrm{NaCl}$, and $5.0 \mathrm{~g}$ Tween-20), 50× TAE (242 g Tris, $57.1 \mathrm{~g}$ acetic acid, $14.61 \mathrm{~g}$ EDTA), $20 \times$ SSC (350.6 g sodium chloride, $176.4 \mathrm{~g}$ trisodium citrate), SDS, and guanidine hydrochloride $(\mathrm{GuHCl})$ solubilizing solution ( 0.1 Tris-HCl, 5 mM EDTA in $6 \mathrm{M} \mathrm{GuCl}, \mathrm{pH}$ 8.0). Antibodies to detect mucins are shown in Table 1, with references. EU-MUC5Ba antibody was a gift from Karine Rousseau (Faculty of Life Sciences, University of Manchester, Manchester, United Kingdom).

\section{Statistics}

Results of relative mucin gene mRNA expression were expressed as means and individual values. Statistical analyses were performed using standardized 2 -tailed $t$ tests. A $P$ value less than 0.05 was considered the minimum acceptable probability for the difference between the means. Mucin concentrations, intensities of mucin immunoreactivity, and osmotic pressure between groups were compared for significance by independent samples 2-tailed $t$ tests using MedCalc statistical software.

\section{Study approval}

All human experimental protocols, including tissue procurement, were approved by the IRB of the University of North Carolina, with the exception of the anonymized CF samples. These were exempted from formal IRB review due to lack of patient identifiers and the intention of being otherwise discarded.

\section{Acknowledgments}

These studies were funded by grants from the Cystic Fibrosis Foundation and from the NIH (CFF RDP R026-CR11, DAVIS07XX0, BUTTON07XX0, BUTTON11G0, KESIME10I0, EHRE07XX0, R01HL103940, P01HL108808, P01 HL110873, P30 DK065988, P50 HL107168, R01 HL092964, and P50HL084934).

Received for publication October 7, 2013, and accepted in revised form April 10, 2014.

Address correspondence to: Mehmet Kesimer, UNC CF Center, 4021 Thurston Bowles, Chapel Hill, North Carolina 275997248, USA. Phone: 919.843.2577; Fax: 919.966.7524; E-mail: mehmet_kesimer@med.unc.edu.
1. Davis PB. Cystic fibrosis since 1938. Am J Respir Crit Care Med. 2006;173(5):475-482.

2. Button $\mathrm{B}$, et al. A periciliary brush promotes the lung health by separating the mucus layer from airway epithelia. Science. 2012;337(6097):937-941.

3. Fahy JV, Dickey BF. Airway mucus function and dysfunction. NEnglJ Med.2010;363(23):2233-2247.

4. Stoltz DA, et al. Cystic fibrosis pigs develop lung disease and exhibit defective bacterial eradication at birth. Sci Transl Med. 2010;2(29):29ra31.

5 . Mott LS, et al. Distribution of early structural lung changes due to cystic fibrosis detected with chest computed tomography. J Pediatr. 2013; 163(1):243-248 e243.

6. Smith JJ, Travis SM, Greenberg EP, Welsh MJ. Cystic fibrosis airway epithelia fail to kill bacteria because of abnormal airway surface fluid. Cell. 1996;85(2):229-236.
7. Pezzulo AA, et al. Reduced airway surface $\mathrm{pH}$ impairs bacterial killing in the porcine cystic fibrosis lung. Nature. 2012;487(7405):109-113.

8. Ganz T. Antimicrobial polypeptides in host defense of the respiratory tract. J Clin Invest. 2002; 109(6):693-697.

9. Puchelle E, Bajolet O, Abely M. Airway mucus in cystic fibrosis. Paediatr Respir Rev. 2002;3(2):115-119.

10. Rose MC, Brown CF, Jacoby JZ, Jacoby JZ 3rd, 
Lynn WS, Kaufman B. Biochemical properties of tracheobronchial mucins from cystic fibrosis and non-cystic fibrosis individuals. Pediatr Res. 1987; 22(5):545-551.

11. Litt M, Khan MA, Chakrin LW, Wardell JR, Wardell JR Jr, Christian P. The viscoelasticity of fractionated canine tracheal mucus. Biorheology. 1974; 11(2):111-117.

12. Hovenberg HW, Davies JR, Carlstedt I. Different mucins are produced by the surface epithelium and the submucosa in human trachea: identification of MUC5AC as a major mucin from the goblet cells. Biochem J. 1996;318(pt 1):319-324.

13. Davies JR, Svitacheva N, Lannefors L, Kornfalt R, Carlstedt I. Identification of MUC5B, MUC5AC and small amounts of MUC2 mucins in cystic fibrosis airway secretions. Biochem J. 1999;344(pt 2):321-330.

14. Matsui $\mathrm{H}$, et al. Evidence for periciliary liquid layer depletion, not abnormal ion composition, in the pathogenesis of cystic fibrosis airways disease. Cell. 1998;95(7):1005-1015.

15. Burgel PR, Montani D, Danel C, Dusser DJ, Nadel JA. A morphometric study of mucins and small airway plugging in cystic fibrosis. Thorax. 2007;62(2):153-161.

16. Henke MO, John G, Germann M, Lindemann H, Rubin BK. MUC5AC and MUC5B mucins increase in cystic fibrosis airway secretions during pulmonary exacerbation. Am J Respir Crit Care Med. 2007;175(8):816-821

17. Henke MO, Renner A, Huber RM, Seeds MC, Rubin BK. MUC5AC and MUC5B mucins are decreased in cystic fibrosis airway secretions. Am J Respir Cell Mol Biol. 2004;31(1):86-91.

18. Henke MO, John G, Rheineck C, Chillappagari S, Naehrlich L, Rubin BK. Serine proteases degrade airway mucins in cystic fibrosis. Infect Immun. 2011;79(8):3438-3444

19. Boat TF, Cheng PW, Iyer RN, Carlson DM, Polony I. Human respiratory tract secretion. Mucous glycoproteins of nonpurulent tracheobronchial secretions, and sputum of patients with bronchitis and cystic fibrosis. Arch Biochem Biophys. 1976;177(1):95-104.

20. Houdret N, et al. Evidence for the in vivo degradation of human respiratory mucins during Pseudomonas aeruginosa infection. Biochimica Biophys Acta. 1989;992(1):96-105.

21. Sagel SD, Chmiel JF, Konstan MW. Sputum biomarkers of inflammation in cystic fibrosis lung disease. Proc Am Thorac Soc. 2007;4(4):406-417.

22. Sagel SD, Wagner BD, Anthony MM, Emmett P, Zemanick ET. Sputum biomarkers of inflammation and lung function decline in children with cystic fibrosis. Am J Respir Crit Care Med. 2012; 186(9):857-865.

23. Thornton DJ, et al. Salivary mucin MG1 is comprised almost entirely of different glycosylated forms of the MUC5B gene product. Glycobiology. 1999;9(3):293-302.

24. Kesimer M, Sheehan JK. Analyzing the functions of large glycoconjugates through the dissipative properties of their absorbed layers using the gelforming mucin MUC5B as an example. Glycobiology. 2008;18(6):463-472.

25 . Houdret N, et al. Evidence for the in vivo degradation of human respiratory mucins during Pseudomonas aeruginosa infection. Biochim Biophys Acta. 1989;992(1):96-105.

26. Matsui H, et al. A physical linkage between cystic fibrosis airway surface dehydration and Pseudomonas aeruginosa biofilms. Proc Natl Acad SciUS A. 2006;103(48):18131-18136.

27. Pearson JP, Pesci EC, Iglewski BH. Roles of Pseudomonas aeruginosa las and rhl quorum-sensing systems in control of elastase and rhamnolipid biosynthesis genes. J Bacteriol. 1997;179(18):5756-5767.

28. Thornton DJ, et al. Identification of a nonmucin glycoprotein (gp-340) from a purified respiratory mucin preparation: evidence for an association involving the MUC5B mucin. Glycobiology. 2001;11(11):969-977.

29. Voynow JA, Gendler SJ, Rose MC. Regulation of mucin genes in chronic inflammatory airway diseases. Am J Respir Cell Mol Biol. 2006;34(6):661-665.

30. Ribeiro CM, et al. Chronic airway infection/inflammation induces a $\mathrm{Ca}^{2+} \mathrm{i}$-dependent hyperinflammatory response in human cystic fibrosis airway epithelia. J Biol Chem. 2005;280(18):17798-17806.

31. Livraghi-Butrico A, et al. Mucus clearance, MyD88-dependent and MyD88-independent immunity modulate lung susceptibility to spontaneous bacterial infection and inflammation. Mucosal Immunol. 2012;5(4):397-408.

32. Hogg JC, et al. The nature of small-airway obstruction in chronic obstructive pulmonary disease. N Engl J Med. 2004;350(26):2645-2653.

33. Cao R, Wang TT, DeMaria G, Sheehan JK, Kesimer M. Mapping the protein domain structures of the respiratory mucins: a mucin proteome coverage study. J Proteome Res. 2012;11(8):4013-4023.

34. Sly PD, et al. Risk factors for bronchiectasis in children with cystic fibrosis. N Engl J Med. 2013; 368(21):1963-1970.

35. Kesimer M, et al. Tracheobronchial air-liquid interface cell culture: a model for innate mucosal defense of the upper airways? Am J Physiol Lung Cell Mol Physiol. 2009;296(1):L92-L100.
36. Kesimer M, Ehre C, Burns KA, Davis CW, Sheehan JK, Pickles RJ. Molecular organization of the mucins and glycocalyx underlying mucus transport over mucosal surfaces of the airways. Mucosal Immunol. 2013;6(2):379-392.

37. Boucher RC. Airway surface dehydration in cystic fibrosis: pathogenesis and therapy. Annu Rev Med. 2007;58:157-170

38. Hays SR, Fahy JV. Characterizing mucous cell remodeling in cystic fibrosis: relationship to neutrophils. Am J Respir Crit Care Med. 2006; 174(9):1018-1024.

39. Feather EA, Russell G. Sputum viscosity in cystic fibrosis of the pancreas and other pulmonary diseases. Br J Dis Chest. 1970;64(4):192-200.

40. Muhlebach MS, Stewart PW, Leigh MW, Noah TL. Quantitation of inflammatory responses to bacteria in young cystic fibrosis and control patients. Am J Respir Crit Care Med. 1999;160(1):186-191.

41. Fulcher ML, Gabriel S, Burns KA, Yankaskas JR, Randell SH. Well-differentiated human airway epithelial cell cultures. Methods Mol Med. 2005; 107:183-206.

42. Holmen JM, et al. Mucins and their O-Glycans from human bronchial epithelial cell cultures. Am J Physiol Lung Cell Mol Physiol. 2004;287(4):L824-L834.

43. Thornton DJ, Gray T, Nettesheim P, Howard M, Koo JS, Sheehan JK. Characterization of mucins from cultured normal human tracheobronchial epithelial cells. Am J Physiol Lung Cell Mol Physiol. 2000;278(6):L1118-L1128.

44. Rousseau K, Wickstrom C, Whitehouse DB, Carlstedt I, Swallow DM. New monoclonal antibodies to non-glycosylated domains of the secreted mucins MUC5B and MUC7. Hybrid Hybridomics. 2003;22(5):293-299.

45. Kesimer M, Sheehan JK. Mass spectrometric analysis of mucin core proteins. Methods Mol Biol. 2012;842:67-79.

46. Thornton DJ, Carlstedt I, Sheehan JK. Identification of glycoproteins on nitrocellulose membranes and gels. Methods Mol Biol. 1994;32:119-128.

47. Jumel K, Fiebrig I, Harding SE. Rapid size distribution and purity analysis of gastric mucus glycoproteins by size exclusion chromatography/multi angle laser light scattering. Int J Biol Macromol. 1996;18(1-2):133-139.

48. Lidell ME, Bara J, Hansson GC. Mapping of the $45 \mathrm{M} 1$ epitope to the C-terminal cysteine-rich part of the human MUC5AC mucin. FEBS $J$. 2008;275(3):481-489.

49. Zhu Y, et al. Munc13-2/- baseline secretion defect reveals source of oligomeric mucins in mouse airways. J Physiol. 2008;586(7):1977-1992. 